\title{
Symplectic integration of hierarchical stellar systems
}

\author{
H. Beust ${ }^{\star}$ \\ Laboratoire d'Astrophysique de Grenoble, Université J. Fourier, BP 53, 38041 Grenoble Cedex 9, France \\ Received 30 September 2002 / Accepted 19 December 2002

\begin{abstract}
Symplectic integration has been used successfully for many years now for the study of dynamics in planetary systems. This technique takes advantage of the fact that in a planetary system, the mass of the central body is much larger than all the other ones; it fails if all massive bodies have comparable masses, such as in multiple stellar systems. A new symplectic integrator is presented that permits the study of the dynamics of hierarchical stellar systems of any size and shape, provided that the hierarchical structure of the system is preserved along the integration. Various application tests of this new integrator are given, such as the gap formation in circumbinary disks, the Kozai resonance in triple systems, the truncated circumbinary disk in the quadruple system GG Tauri, and the dynamics of the sextuple system Castor.
\end{abstract}

Key words. methods: $N$-body simulations - methods: numerical - celestial mechanics - stars: binaries: general - stars: circumstellar matter

\section{Introduction}

Symplectic integrators have become very popular in the last decade in planetary dynamics problems, as they present two major advantages with respect to other $n$-body integrators: First, they exhibit no long-term accumulation of energy error, and second they provide a gain of at least one order of magnitude in computation speed, for equivalent accuracy, because they allow one to adopt a much larger time-step than other integrators for the same result, leading to a much more rapid integration. The most popular symplectic integrator is the secondorder MVS (Mixed Variable Symplectic) one, first described by Wisdom \& Holman (1991) and implemented by Levison \& Duncan (1994) in their SWIFT_MVS integration package.

The general assumption for efficient symplectic integrators is that the the motion of each body is separated into a dominant Keplerian part, solved to machine precision with an appropriate algorithm, and a perturbation that is solved numerically. In planetary dynamics, the dominant Keplerian part is due to the central star, and the perturbations to the planets. The MVS integrator is based on this assumption. However, the integration is no longer valid whenever the perturbation becomes larger than, or even of comparable order to the dominant Keplerian part. In planetary dynamics, this occurs when some of the bodies get close to each other, i.e., in the so-called case of close encounters. A large effort was done in the past years to make symplectic integrators handle close encounters (Levison \& Duncan 1994; Chambers 1999; Duncan et al. 1998): The basic idea is to reduce the integration time-step during close encounters, but this necessarily no longer preserves the symplectic character

\footnotetext{
* e-mail: Herve.Beust@obs.ujf-grenoble.fr
}

of the integration, although from a statistical point of view, the results are still valid (Levison \& Duncan 1994). Chambers (1999), in his MERCURY integrator, incorporates the close encounter perturbation into the Keplerian term during close encounters, but with the consequence of a significant decrease of the integration speed. Finally, Duncan et al. (1998), in their SyMBA integrator, use a multiple time-step technique that handles close encounters while remaining symplectic. However, it fails in integrating close encounters with the central star. A variant to this method is presented in Levison \& Duncan (2000) that overcomes this difficulty but is more CPU-time consuming.

In planetary dynamics, the motion of the bodies is nearly Keplerian far from close encounters because the mass of the central star largely overcomes that of the other bodies. This is no longer valid in multiple stellar systems, where several massive centers can be present. Multiple stellar systems can be classified into two sets: Trapezian and Hierarchical systems. In trapezian systems, all relative distances between the bodies are more or less comparable. These systems are usually unstable on time-scales of a few Myrs or less, and it is very difficult in general to identify a dominant Keplerian part in the relative motion of their components. Hierarchical systems are conversely characterized by nested orbits of very different sizes, and are usually more stable. In that case, the motion of the bodies remains nearly Keplerian thanks to the very different size of the nested orbits, although all massive bodies may have comparable masses.

However, unless in specific cases, the MVS technique cannot be used directly to compute the dynamics of hierarchical multiple stellar system, as it needs to hold one of the bodies as the "Sun", and the other ones as the perturbing "planets". 
An interesting new technique dedicated to the study of planets in binary systems was recently presented by Chambers et al. (2002), with application to the $\alpha$ Centauri system (Quintana et al. 2002). This integrator is however limited to binary systems only. The purpose of this paper is to present a new MVSlike integrator dedicated to the integration of the dynamics of hierarchical systems of any size and structure, provided the hierarchy is preserved. We give this new method the name HJS (Hierarchical Jacobi Symplectic). In Sect. 2, we briefly review the theory of symplectic integrators and the MVS method, and develop the theory for the HJS method. Various integration tests of growing complexity are given in Sect. 3. Our conclusions are presented in Sect. 4.

\section{Theory of the Hierarchical Jacobi symplectic integrator}

\subsection{Symplectic integration}

An integration of any Hamiltonian system is symplectic when it exactly preserves the generalized areas in phase-space. This is in particular the case if the integration exactly solves (within computer round-off errors) a Hamiltonian. Symplectic integrators do not actually strictly solve the real Hamiltonian of the problem, but do it for a surrogate Hamiltonian assumed to be close to the real one. Once this is achieved it can be shown that there is no long-term drift of the energy. This is not the case for more classical methods (Runge-Kutta, Burlish \& Stoer...) used in celestial mechanics, that are far from being symplectic.

The theory of symplectic integration is based on the Hamilton equations of motion that apply to any $n$-body problem. Its background is for example described in Saha \& Tremaine (1992) and Chambers (1999). The key idea of symplectic integration is to split the Hamiltonian $H$ the problem into pieces, say

$H=H_{A}+H_{B}$,

each of them being exactly integrable within computer roundoff errors. If this is possible, then a first order symplectic integrator can be build if, starting from the generalized positions and momenta at time $t-\tau$, we derive their values at time $t$ by

1. advancing $H_{B}$ by a time step $\tau$;

2. advancing $H_{A}$ by the same amount.

Of course, we get another integrator if we first advance $H_{A}$ and then $H_{B}$. The integration is of first order because it exactly solves a surrogate Hamiltonian $\tilde{H}$ that is close to $H$ within $O(\tau)$. Hence a full integration step consists of two separate sub-steps.

A second order integrator can be achieved if we combine now three sub-steps:

1. advance $H_{B}$ by a half step $\tau / 2$;

2. advance $H_{A}$ for the full time step $\tau$;

3. Re-advance $H_{B}$ by $\tau / 2$.

In that case now, the surrogate Hamiltonian $\tilde{H}$ is close to $H$ within $O\left(\tau^{2}\right)$. The common MVS integrator is based on this second order scheme. Our basic HJS integrator will also be based on this scheme, but the way we split $H$ into $H_{A}+H_{B}$ will be different. Higher order (fourth, sixth... ) schemes can be build by combining more sub-steps (Yoshida 1990; Chambers \& Murison 2000), but they are less easy to implement, so that the second order method has become by far the most commonly used. As a matter of fact, it provides accurate results for most problems in planetary dynamics. For comparison, we nevertheless implemented for the HJS method two distinct integrators: a second order one called HJS2 following the 3 sub-steps scheme given above, and a fourth order one using 7 sub-steps (quoted as S4B by Chambers \& Murison 2000), that we call HJS4. Whenever not specified, we use in the following the second order method.

Additional accuracy is reached in symplectic integration if we have now in addition $H_{B} \ll H_{A}$, i.e., $H_{B}=\epsilon H_{A}$ with $\epsilon \ll 1$. In that case we have

$\tilde{H}=H+O\left(\epsilon \tau^{2}\right)$.

In practice, this last property allows us to integrate with much longer time-steps than with conventional methods. $H_{B} \ll H_{A}$ is then a important condition to ensure for the validity of the integration. In classical celestial mechanics problems, $H_{A}$ will by the dominant Keplerian interaction and $H_{B}$ will be the perturbation.

\subsection{The MVS method (Wisdom - Holman mapping)}

Let us consider a general $n$-body gravitational system, with bodies labeled from 1 to $n$, masses $m_{1}, \ldots, m_{n}$, positions $\boldsymbol{x}_{1}, \ldots, \boldsymbol{x}_{n}$ and impulsions $\boldsymbol{p}_{1}, \ldots, \boldsymbol{p}_{n}$ in the barycentric referential frame. Then the Hamiltonian of the system reads

$H=\sum_{i=1}^{n} \frac{p_{i}^{2}}{2 m_{i}}-\sum_{1 \leq i<j \leq n} \frac{G m_{i} m_{j}}{r_{i j}}$,

where $G$ is the constant of gravitation and $r_{i j}=\left\|\boldsymbol{x}_{j}-\boldsymbol{x}_{i}\right\|$ is the distances between bodies $i$ and $j$. Wisdom \& Holman (1991) introduce the classical Jacobi coordinates $\boldsymbol{x}_{i}^{\prime}(i=1 \ldots n)$, defined as follows:

$\boldsymbol{x}_{1}^{\prime}=\boldsymbol{r}_{n}$

$\boldsymbol{x}_{i}^{\prime}=\boldsymbol{x}_{i}-\boldsymbol{r}_{i-1}$ for $2 \leq i \leq n$,

where $\boldsymbol{r}_{i}$ is the location of the center or mass of bodies up to $i$. Wisdom \& Holman (1991) rewrite the Hamiltonian (3) as

$H=\frac{p_{1}^{\prime 2}}{2 m_{1}^{\prime}}+\sum_{i=2}^{n} \frac{p_{i}^{\prime 2}}{2 m_{i}^{\prime}}-\sum_{i=2}^{n} \frac{G m_{i} m_{1}}{r_{i 1}}-\sum_{2 \leq i<j} \frac{G m_{i} m_{j}}{r_{i j}}$,

where $\boldsymbol{p}_{i}^{\prime}=m_{i}^{\prime}\left(\mathrm{d} \boldsymbol{x}_{i}^{\prime} / \mathrm{d} t\right)$ is the conjugate momentum of $\boldsymbol{x}_{i}^{\prime}$, and with

$m_{i}^{\prime}=\frac{\eta_{i-1}}{\eta_{i}} m_{i} \quad$ where $\quad \eta_{k}=\sum_{j=1}^{k} m_{i}$.

This holds for $i>1$. For $i=1, m_{1}^{\prime}$ is the total mass of the system.

In Eq. (6), Wisdom \& Holman (1991) particularize the role of the first body \#1, which appears natural in the case of a planetary system (body \#1 is the "Sun"). $H$ appears not to depend 
on the first Jacobi coordinate $\boldsymbol{x}_{1}$, showing as expected that the center of mass moves as a free particle. Actually, if the origin of the referential frame is set to the center of mass of the system, then we have $\boldsymbol{x}^{\prime}{ }_{1}=0$. The first term of Eq. (6) is then a constant and can be removed from the expression of $H$. Wisdom \& Holman (1991) then add and subtract the term

$\sum_{i=2}^{n} \frac{G m_{i} m_{1}}{r_{i}^{\prime}}$

and split the Hamiltonian as follows:

$H_{A}=\sum_{i=2}^{n}\left(\frac{p_{i}^{\prime 2}}{2 m_{i}^{\prime}}-\frac{G m_{i} m_{1}}{r_{i}^{\prime}}\right)$;

$H_{B}=\sum_{i=2}^{n}\left(\frac{G m_{i} m_{1}}{r_{i}^{\prime}}-\frac{G m_{i} m_{1}}{r_{i 1}}\right)-\sum_{2 \leq i<j} \frac{G m_{i} m_{j}}{r_{i j}}$.

$H_{A}$ is an independent sum of $n-1$ pure Keplerian Hamiltonians in Jacobi coordinates, each one corresponding to body \#i orbiting the central body \#1 without any perturbation from the other ones. It is thus integrable. $H_{B}$ is the perturbation Hamiltonian that only depends on the position variables $\boldsymbol{x}_{i}{ }_{i}(2 \leq i \leq n)$. It is thus integrable straightforwardly.

The MVS method is entirely based on this splitting of the Hamiltonian. It takes advantage of the fact that usually in a planetary system, we have indeed $H_{B} \ll H_{A}$. In order for this to be ensured, two conditions must be fulfilled: i) for each $i>1$, we must have $m_{i} \ll m_{1}$, and ii) the terms $1 / r_{i j}$ should not be abnormally large. If this is the case, then the second term of $H_{B}$ is obviously small with respect to $H_{A}$ within the mass ratios of the planets to the Sun, as well as the first term, because $r_{i}^{\prime} \approx r_{i 1}$.

In the case of a close encounter, one of the $1 / r_{i j}$ terms may become large, and the condition $H_{B} \ll H_{A}$ is no longer ensured. As mentioned in the Introduction, a large effort was made in recent years to overcome this difficulty (Levison \& Duncan 1994; Chambers 1999; Duncan et al. 1998).

\subsection{Hierarchical stellar systems and the HJS (Hierarchical Jacobi Symplectic) method}

In the present study, we will not be concerned by close encounters, but rather by a failure of the first condition that makes $H_{B} \ll H_{A}$. As a matter of fact, in a multiple stellar system, the masses of the various bodies may be comparable. Consequently, integrating a hierarchical system with the conventional MVS technique, even holding the largest body as the "sun", may lead to an immediate failure, unless the time-scale chosen is very short. As described below, there are some very simple cases where the MVS technique can still handle multiple systems, but it cannot be generalized to any configuration. It is nevertheless possible to design an integration scheme valid for hierarchical systems of any shape that preserves all the desired properties of the symplectic integration. To do this, we need to change the way of splitting the Hamiltonian with respect to Eqs. (8) and (9). We also need to define a new set of coordinates that appear as a generalization of the Jacobi coordinates.
The Jacobi coordinates are indeed well suited to describe a hierarchical system, as they describe the location of each body with respect to the center of mass of the bodies below it. However, this description holds only if the system assumes a "linearly" hierarchical structure, where the first orbit is the smallest one, which is nested in the second one, itself nested in the third one, etc. All hierarchical systems do not strictly assume such a structure. For example, a quadruple system consisting of two binaries orbiting each other (the double-binary case) obviously does not obey this rule, as we have two independent orbits orbiting each other. The hierarchical structure of this system is rather tree-like than "linear". In order to build an integration scheme valid for every hierarchical structure, we need to introduce a generalization of the standard Jacobi coordinates.

Our $n$-body system is still described by the positions and impulsions of the $n$ bodies in the barycentric referential frame, still noted $\boldsymbol{x}_{i}$ and momenta $\boldsymbol{p}_{i}$ as above. In a general manner, we introduce $n-1$ Jacobi-like coordinates $\boldsymbol{x}_{i}^{\prime}(i=2 \ldots n)$, each one corresponding to the location of the center of mass of a given subset of the bodies with respect to the center or mass of another, distinct, subset of the bodies. We also define as above $\boldsymbol{x}_{1}{ }_{1}$ as the location of the center of mass of the system (i.e. $\boldsymbol{x}_{1}^{\prime}=0$ with a convenient choice of origin). It is equivalent to say that we describe our system with $n-1$ "orbits", each one corresponding to the center of mass of a first subset of the bodies orbiting the center of mass of a second subset of the bodies. In the following, we shall refer for each orbit to the first subset as the "satellites" of the orbit, and to the second subset as the "centers" of the orbit. For each orbit $k$, we define

$$
\begin{aligned}
\eta_{k} & =\sum_{i, \text { centers of } k} m_{i}, \\
\mu_{k} & =\sum_{i, \text { satellites of } k} m_{i} .
\end{aligned}
$$

The generalized Jacobi coordinate $\boldsymbol{x}^{\prime}{ }_{k}$ is then defined as

$\boldsymbol{x}_{k}^{\prime}=\sum_{i \text {, satellites of } k} \frac{m_{i} \boldsymbol{x}_{i}}{\mu_{k}}-\sum_{i, \text { centers of } k} \frac{m_{i} \boldsymbol{x}_{i}}{\eta_{k}}$.

If the system assumes a linear structure, with bodies labeled from the interior to the exterior, then our coordinate system is the classical Jacobi coordinate system.

We may give a matrix formulation of this transformation. We define $\boldsymbol{X}$ as the $n$-vector of the barycentric positions of the bodies, and $\boldsymbol{X}^{\prime}$ as the $n$-vector of the generalized Jacobi positions. Equation (12) for each $k$ can be viewed as $\boldsymbol{X}^{\prime}=M \boldsymbol{X}$, where $M$ is a fixed $n \times n$ matrix, defined by

1. $M_{1 j}=m_{j} / m$ (if $m$ is the total mass of the system),

2. $M_{i j}=m_{j} / \mu_{i}$ if body \# $j$ is a satellite in orbit \# $i$,

3. $M_{i j}=-m_{j} / \eta_{i}$ if body \# $j$ is a center in orbit \#i,

4. $M_{i j}=0$ otherwise.

The transformation $\boldsymbol{X} \longrightarrow \boldsymbol{X}^{\prime}$ easily extends to a canonical transformation of the conjugate momenta $\boldsymbol{P} \longrightarrow \boldsymbol{P}^{\prime}$, by

$\boldsymbol{P}^{\prime}={ }^{t} M^{-1} \boldsymbol{P}$.

Of course, in order to be valid, our transformation must obey some rules dictated by the hierarchical structure of the system. 
More specifically, given one orbit $i$ with its sets of centers and satellites, there can be only three kinds of other orbits (apart from the first dummy orbit corresponding to the center of mass of the system):

1. Foreign orbits, i.e., orbits with centers and satellites fully distinct from the centers and satellites of orbit $i$;

2. Inner orbits, i.e., orbits with centers and satellites all taken in one of the two sets (centers or satellites) of orbit $i$;

3. Outer orbits, i.e., orbits for which all centers and satellites of orbit $i$ fall in one of the two subsets of the orbit we are considering.

It is equivalent to say that given two orbits, either they are foreign, or one is inner (resp. outer) to the other one. For example, in the double-binary system introduced above, we may define orbit \#2 as the orbit of the first binary, orbit \#3 as the orbit of the second binary, and orbit \#4 as the relative orbit between the two binaries. Orbits \#2 and \#3 are clearly inner to orbit $\# 4$, while they are foreign to each other. If this structure is respected (and only in this case), then it is possible to show (see Appendix A for details) that the kinetic energy of the system may be rewritten as

$$
\sum_{\text {bodies } i=1}^{n} \frac{p_{i}^{2}}{2 m_{i}}=\sum_{\text {orbits } k=2}^{n} \frac{p_{k}^{\prime 2}}{2 m_{k}^{\prime}},
$$

where the reduced mass for each orbit is

$m_{k}^{\prime}=\frac{\eta_{k} \mu_{k}}{\eta_{k}+\mu_{k}}$

We have implicitly assumed that $\boldsymbol{x}_{1}{ }_{1}=0$. We also add the convention $m_{1}^{\prime}=m$.

Hence as for the barycentric coordinates, in the generalized Jacobi coordinates, the kinetic energy appears as a weighted sum of the squared momenta (there are no cross terms of the form $\left.\boldsymbol{p}_{i}{ }_{i} \boldsymbol{p}^{\prime}{ }_{j}\right)$. It is equivalent to say that $\boldsymbol{p}_{i}{ }_{i}=m_{i}^{\prime} \mathrm{d} \boldsymbol{x}_{i}{ }_{i} / \mathrm{d} t$ for each $i$. This property is the cornerstone of our method. It allows the Hamiltonian to naturally split, as for the MVS method, into a sum of separate Keplerian Hamiltonians plus a perturbation that does not depend on the momenta. This is indeed the reason why Wisdom \& Holman (1991) make use of the Jacobi coordinates in the Keplerian part of their method, even though everything else is computed in heliocentric coordinates. In their Democratic Heliocentric method, developed for integrating close encounters, Duncan et al. (1998) use heliocentric coordinates. In that case, the kinetic energy does not reduce to such a simple form. It is then necessary to split the Hamiltonian into three separate parts. This does not cause big trouble except for orbits with very small perihelia. A special modification of the method has been developed in Levison \& Duncan (2000) to overcome this difficulty.

With the kinetic energy expressed as in Eq. (14), we may now split the Hamiltonian (3) as

$$
\begin{aligned}
& H_{A}=\sum_{k=2}^{n}\left(\frac{p_{k}^{\prime 2}}{2 m_{k}^{\prime}}-\frac{G \mu_{k} \eta_{k}}{r_{k}^{\prime}}\right) \\
& H_{B}=\sum_{k=2}^{n} \frac{G \mu_{k} \eta_{k}}{r_{k}^{\prime}}-\sum_{1 \leq i<j \leq n} \frac{G m_{i} m_{j}}{r_{i j}} .
\end{aligned}
$$

If the system assumes the hierarchical structure quoted above, and if we assume that the nested orbits are of very different sizes (which is usually the case for hierarchical systems), it is possible to show that $H_{B} \ll H_{A}$ (see Appendix B for details). It is important to note that this result arises without making any hypothesis about the masses of the various bodies. In the MVS method (Eqs. (8) and (9)), $H_{B} \ll H_{A}$ because the first mass $\left(m_{1}\right)$ is much larger than all the others. Here, all masses may be comparable or not; this does not affect the result.

Both methods have distinct application fields. In the Solar System, there is only a factor $\sim 2$ between two successive planetary orbits. The stability of the system is ensured by the mass of the Sun. Hence the MVS method is well suited in this case. In hierarchical stellar systems, there is no dominant mass, but the scaling factor between successive orbits is usually much larger than 2 .

Our symplectic method is entirely based on this splitting of the Hamiltonian. In fact, this is the natural splitting of generalized Jacobi variables. As a result, we call our method the Hierarchical Jacobi Symplectic (HJS).

A special case arises if we add some massless particles, usually called "test particles". In a stellar system, the hierarchical structure is defined from the relative orbits of the massive bodies. Adding one test particle means adding a body, but also an orbit. The "satellites" of this orbit reduce to the particle itself, but the centers, i.e., the subset of bodies the particle is intended to orbit around, must be specified. Moreover, even if this new massless body does not affect the massive ones, the new orbit must follow the hierarchical structure of the system. In practice, this means that the test particle has to be added to the list of centers or satellites of some outer orbits. This of course does not affect the dynamics of the other bodies, but does for the particle itself. It adds some terms to the second order equations derived from $H_{B}$ (and used in the code) that define the perturbing acceleration on the particle. For example, we consider the double-binary system in which we want to add a circumbinary disk of test particles orbiting one of the binaries. Each of these particles will be considered as orbiting the center of mass of that binary, but it must be considered as an additional center for the relative orbit between the two binaries, otherwise the hierarchical rules are not fulfilled. Of course this does not affect the dynamics of the outer orbit, but in practice, this adds a term to the dynamics of each particle.

In the following, we apply the HJS method in various situations of astrophysical interest relevant to the dynamics of multiple stellar systems. The HJS method applies indeed to a large variety of situations. The limitations of the method are those that ensure the condition $H_{B} \ll H_{A}$ : i) close encounters must be avoided, ii) the hierarchy must not change: a given particle orbiting a subset of centers must keep orbiting around the same centers during the computation. In the runs we describe below, these conditions are not strictly speaking respected for the test particles, as gravitational perturbations may cause a lot of particles to escape from the system. However, we are mainly interested in those particles that remain in the system. The fact that the dynamics of the particles that escape are not computed with great accuracy at ejection time does not affect our conclusion, the important thing being that the particles initially located at 
some locations in the system are actually unstable, irrespective of their dynamics at ejection time.

\section{Application tests for the HJS integrator}

Designing accurate tests for the HJS method is not a straightforward task, as there is virtually no typical behavior for multiple stellar systems, each configuration being in itself a peculiar case. There are basically three kinds of features that can be tested:

The most straightforward test is to investigate for any integration the stability of the global energy and of the angular momentum of the system over the integration time. Note that this test is relevant only if more than two massive bodies are present, independent of the number of test particles. If this is not the case, then the relative orbit between the two bodies is fixed, and the method solves it obviously to machine precision.

Second, any integration can be performed in parallel with a more conventional, and more CPU-time consuming integrator like the Burlish \& Stoer method. However the comparison will be of interest only over rather short time spans, as the Burlish $\&$ Stoer method is not symplectic.

Third, there are some known results concerning peculiar dynamical situations that we aim to reproduce (but more rapidly) with the HJS integrator. We must obtain stability when it is expected. This may concern test particles (e.g., gaps in circumbinary disks), or the massive bodies themselves, such as the Kozai resonance in triple systems. This will constitute our guideline for the following tests.

\subsection{Circumbinary disks}

Many young stellar systems were observed in recent years to harbor dusty or gaseous disks orbiting a binary star. These disks are today known as circumbinary disks. This includes the examples of GG Tauri (Guilloteau et al. 1999; White et al. 1999), UY Ori (Duvert et al. 1998; Close et al. 1998), and HD 98800 (Koerner et al. 2000). Circumbinary disks are known to be cleared from inside by interaction with the components of the binary, leading to a sharp inner edge located well outside the orbit of the central binary. There is observational evidence for this fact (Guilloteau et al. 1999), and this was predicted by many theoretical studies, often using smoothed particle hydrodynamics (SPH) (Artymowicz \& Lubow 1994; Artymowicz \& Lubow 1996; Bate 2000). All these simulations show that the inner gap of the disk opens within a few orbital periods of the binary, with spiral density waves extending far in the remaining disk. Artymowicz \& Lubow (1996) show that some of the tidally eroded mass can flow towards the individual stars by so-called "streamers".

Trying to reproduce these characteristics is a valuable test for the HJS integrator. In fact, a circumbinary disk can still be simulated with the standard MVS integrator if we give the test particles the higher indexes. One of the two stars needs to be taken as the "sun", the other one being a "perturber". This still works because there are here only two massive bodies; their mutual orbit is then left unchanged and is solved exactly independent of the method. However we must assume that the dominant Keplerian motion of the test particles is around the "sun" chosen for the system. Hence the perturbation arising from the other star is comparable to the dominant Keplerian motion, but it still works because we adopt a time-step of $1 / 20$ of the smallest orbital period, i.e., that of the binary, which represents a much smaller fraction of the orbital periods of the test particles.

However, the orbital motion of test particles in a circumbinary disk is usually described with respect to the center of mass of the binary, which is exactly the framework of the HJS method. As a matter of fact, the HJS formulation appears much more natural for such a system. In an HJS integration, the circumbinary disk is simulated as a set of test particles orbiting the center of mass of a binary star (to which is given the total mass of the binary), and perturbed by each component. The main goal is to reproduce the inner gap clearing phase, in both size and shape. We nevertheless do not expect to properly simulate the streamers onto the central stars, as whenever particles fall in between the two stars, or close to one of them, it can hardly be regarded as mainly orbiting the center of mass of the system. As the particles do not interact with each other, we cannot give any viscosity to the disk, contrary to SPH integrations. However, with respect to SPH simulations, we are able to take many more particles in the disk, and to integrate the dynamics of the disk over a much longer time span, which allows us to investigate the relaxation of the disk and the dissipation of the spiral density waves.

Dynamically speaking, a binary system is characterized by an orbital eccentricity $e$ and by a mass parameter $\mu$ : the masses of the stars are $\mu M$ and $(1-\mu) M$ where $M$ is the total mass. The semi-major axis $a$ of the binary does not need to be specified, as the whole problem (gap sizes, evolution time-scale) just rescales with $a$. Figure 1 shows the evolution of the gap for a disk orbiting a binary with $(e=0.1, \mu=0.3)$, as computed with the HJS2 (second order) integrator. All distances are given relative to $a$. The initial disk holds $10^{5}$ particles located between $r=1.5 a$ and $r=3.5 a$ from the center of mass of the system, with a surface density $\propto r^{-1}$. Note that the inner edge of the initial disk is far inside the expected size of the gap, so that it has no influence on the result. The particles are randomly chosen with eccentricities between 0 and 0.1 and inclinations with respect to the binary orbital plane ranging between 0 and $3^{\circ}$.

Figure 1 must be compared to Fig. 9 from Artymowicz \& Lubow (1994) which displays the evolution of a similar system, computed with SPH. We see in Fig. 1 that we reproduce the gap clearing phase, the spiral density waves and even the streamers. However, we may not trust the dynamics of the particles once they have fallen between the two stars. This does not matter, as in any case, these particles are rapidly removed from the system (or accreted by the stars). The final gap is at $r=2.6 a$, consistent with the simulations of Artymowicz \& Lubow (1994).

The time-step used in our simulation is $1 / 20$ of the orbital period of the binary. This is a classical choice for MVS-like integrators. Any other, non-symplectic integrator would need to use a time-step at least 50 times less to get the same result. As a matter of fact, we were able to carry out the integration up to $10^{5}$ orbital periods (this took approximatively 1 day of CPUtime), which is not possible with SPH. At $t=10^{5}$, the gap is totally cleared, and the sharp inner edge of the disk clearly 

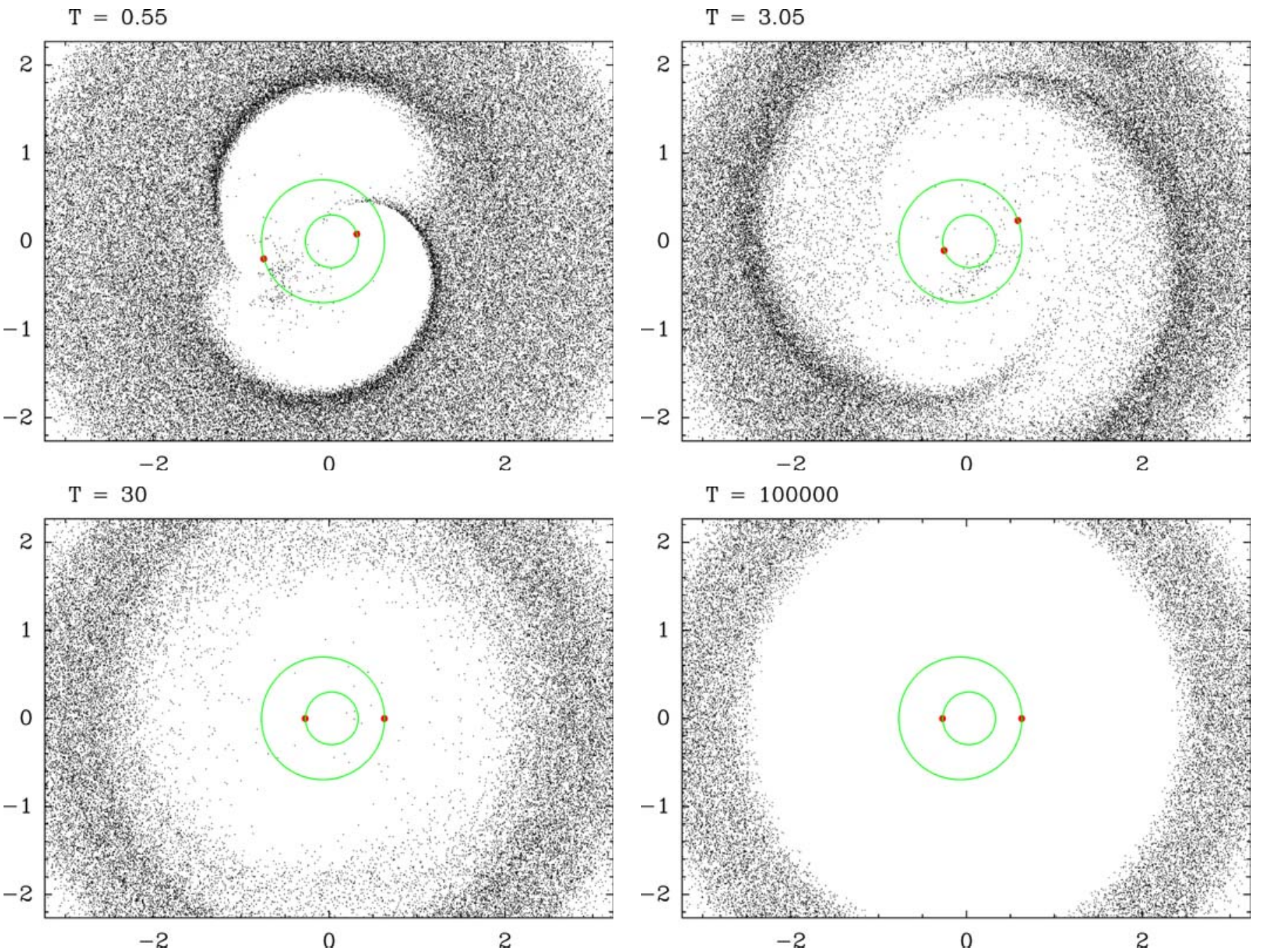

Fig. 1. Dynamical evolution of a circumbinary disk orbiting a binary with mass parameter $\mu=0.3$, and orbital eccentricity $e=0.1$. The axes are labeled in units of the semi-major axis of the binary. For each snapshot, the time is given above the plot in units of the orbital period of the binary. The stars themselves are indicated as filled circles, together with their orbits in a barycentric referential frame.

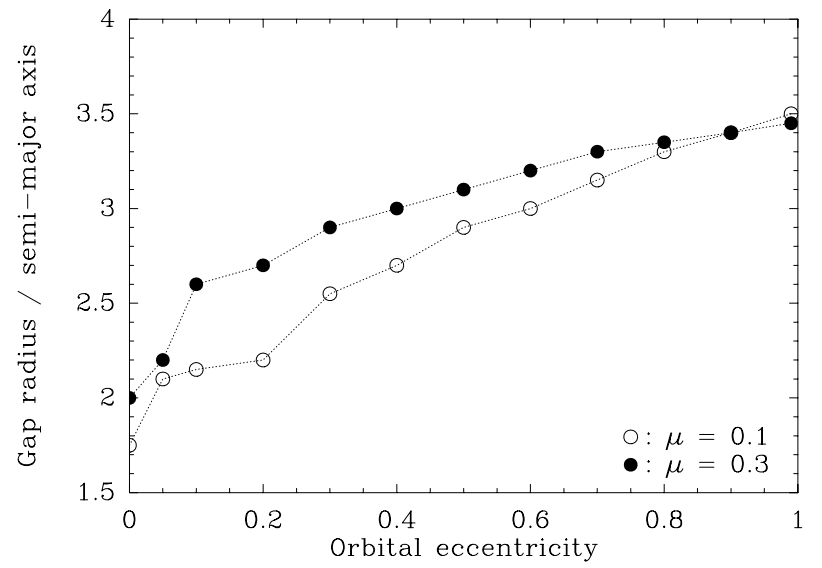

Fig. 2. Approximate radius of the inner edge of the circumbinary disk as computed with HJS integrations, in units of the semi major axis $a$ of the binary, for various values of $e$ and $\mu$. The radius of the gap is appreciated at $t=300$ orbital periods of the binary.

shows up. In fact, at this time, the remaining circumbinary disk is fully stable and relaxed. The disk does no longer lose particles. Actually the gap clearing is almost achieved after a few hundred periods.

We performed several simulations of the same kind, for various sets of values $(e, \mu)$. The results are summarized in Fig. 2 which displays the size of the computed inner gap of the circumbinary disk as a function of the eccentricity $e$, for two values of $\mu$. The size of the gap is appreciated after 300 orbital periods of the binary. The result are consistent with the corresponding figures from Artymowicz \& Lubow (1994). We note as expected a gradual increase of the gap as a function of the eccentricity, and on average a larger gap for a larger $\mu$. The sudden increase of the gap when reaching $r \simeq 2.2 a$ is due to the $4: 1$ commensurability that falls at $r=2.52 a$. This commensurability always clears a ring-like additional gap; whenever the inner edge of the disk gets close to this gap, it merges with it, leading to a sudden increase of the central gap.

Apart from a circumbinary disk, a binary star can also harbor circumstellar disks orbiting each of its components. Of course we expect these disks, if present, to be tidally truncated outwards. This is what is actually observed in the HK Tauri system (Koresko 1998; Stapelfeldt et al. 1998). In this system, 
$\mathrm{T}=0.5$

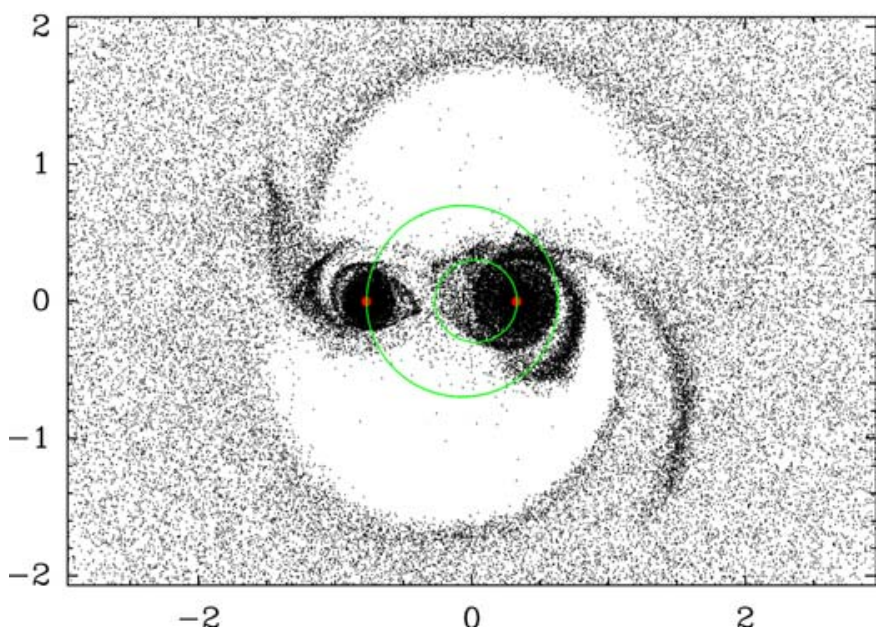

$\mathrm{T}=100$

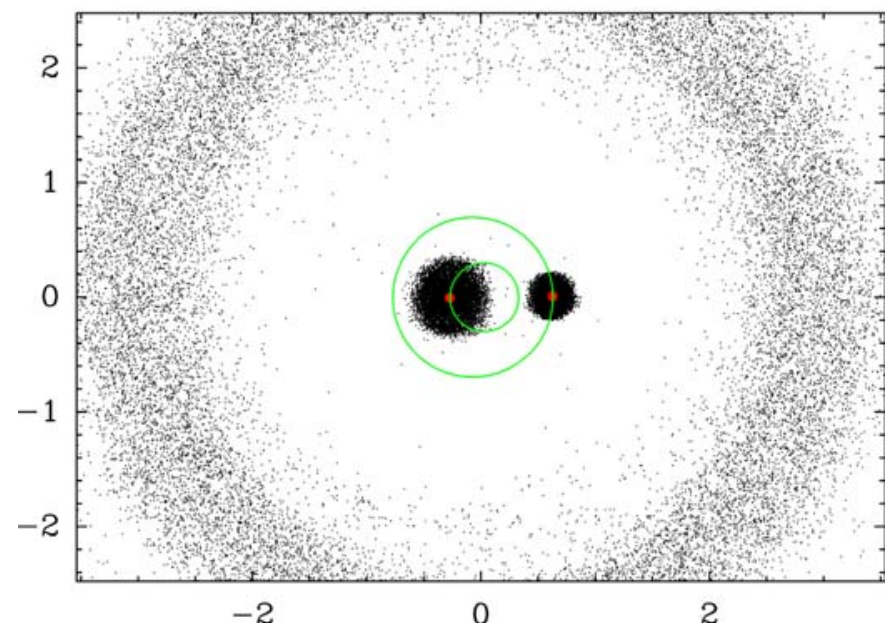

Fig. 3. Dynamical evolution of the same system as in Fig. 1, but to which two circumstellar disks orbiting the individual stars have been added. The plotting conventions are the same as for Fig. 1.

the secondary star was observed to be surrounded by a circumstellar disk, almost seen edge-on from the Earth. This disk is probably tidally truncated by the primary, as it extends to $\sim 1 / 3$ of the projected separation of the two stars Stapelfeldt et al. (1998).

In order to further test the HJS method, we added to the previous simulation two circumstellar disks of particles orbiting each individual star. More precisely, we still take $10^{5}$ particles, but we leave 50000 of them in the circumbinary dusk, and put 25000 in two circumstellar disks orbiting each star. The circumstellar disks are initially chosen identical, with an initial outer radius of $0.7 a$. These disks are assumed for simplicity to be coplanar with the binary orbit and the circumbinary disk, but inclined disks, as this seems to be the case for HK Tauri (Koresko 1998), could be easily simulated. Note that such a configuration can no longer be simulated with the MVS method, as now all particles do not orbit the same massive center.

Figure 3 displays two snapshots of that simulation, at $t=0.5$ orbital period of the binary, and $t=100$ periods. In the first snapshot, we clearly see the tidal erosion of the outer edges of the individual disks. The sculpting of the inner edge of the circumbinary disk is the same as in Fig. 2. In the second snapshot, the disks have stabilized. We see the sharp truncation of the disks. As expected, the disk surrounding the secondary star is smaller than the one orbiting the primary star (recall that the disks were initially identical). The remaining disks do not exhibit any strong tidal perturbation by the companion star, as this seems to be also the case for HK Tauri (Koresko 1998).

\subsection{Triple stellar systems}

Hierarchical triple systems consist usually of three bodies of comparable masses, the distance between two of them being much smaller than their distance to the third body. In a first approximation, the dynamics of such a system can be regarded as two nested Keplerian orbits, the first one involving a close binary (the two first bodies), and the second one describing the third body orbiting the center of mass of the close binary. We shall hereafter refer the orbit of the close binary as the inner orbit, and the orbit of the third body as the outer orbit.

Of course, in the framework of the HJS formalism, this description with two fixed orbits corresponds exactly to the Hamiltonian $H_{A}$ given by Eq. (16), and the two orbits themselves correspond to the Jacobi coordinates introduced above. Due to the perturbing Hamiltonian $H_{B}$ (Eq. (17)), the two orbits are slowly modified. We expect the HJS integrator to efficiently compute this secular evolution. Here again, the MVS integrator can still work for such a system, because the description of the inner orbit is the same in both formulations. The outer orbit is however not described identically in both method, but as the time-step is fixed by the inner one, resulting in a very small fraction of the outer orbital period, the dynamics of the third body can still be computed with the MVS technique, but less safely than if we adopt the more natural hierarchical formulation of HJS.

The global behavior of such triple systems has been the subject of several theoretical investigations in the past 30 years (Harrington 1968; Mazeh \& Shaham 1977, 1979; Söderhjelm 1984; Krymolowski \& Mazeh 1999). First, the semi-major axes of both orbits appear very stable, apart from very small amplitude and short period variations. This is due to the fact that the short period terms rapidly average over a few orbits; second, the outer orbit appears very stable, apart from a slow precession of the line of apsides. The most striking fact concerns the evolution of the inner orbit. Despite a constant semi-major axis, its eccentricity can be subject to large modulations, even if the orbit is initially circular, the main parameter controlling this being the mutual inclination between the two orbits. This was initially described by Harrington (1968), Mazeh \& Shaham (1977, 1979), and Söderhjelm (1982).

A full numerical and semi-analytical study is given in Krymolowski \& Mazeh (1999). Here we aim to reproduce their results using the HJS integrator. In order to make the comparison possible, we then assume the same initial conditions as them, i.e. a mass ratio $q_{\text {in }}=m_{1} / m_{2}=0.27$ between the two components of the inner binary, and similarly 


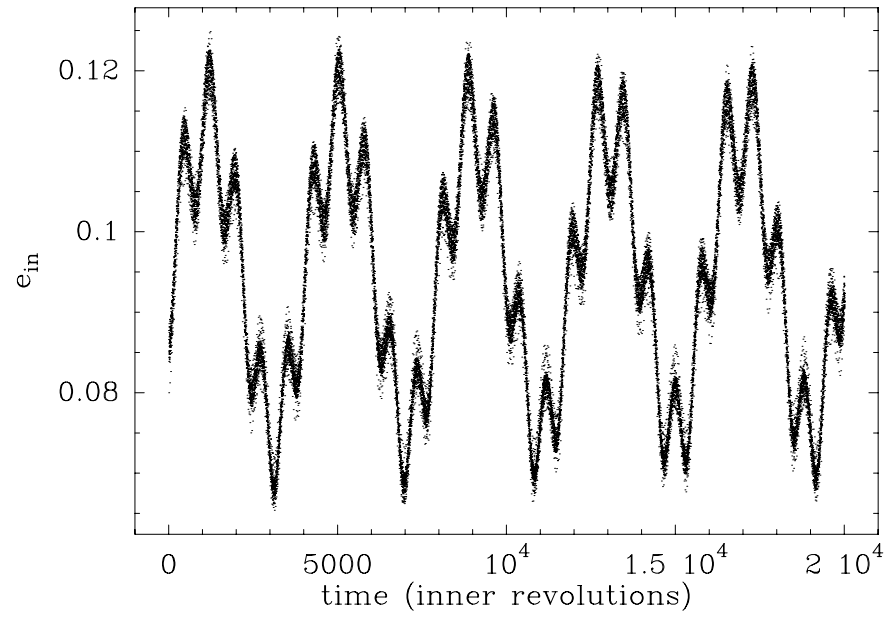

Fig. 4. Evolution of the eccentricity $e_{\text {in }}$ of the inner orbit of the triple system (see text), as computed with the HJS integrator, for an initial mutual inclination $i=20^{\circ}$. The evolution is computed over $2 \times 10^{4} \mathrm{rev}-$ olutions of the inner orbit.

$q_{\text {out }}=m_{3} /\left(m_{1}+m_{2}\right)=0.44$ for the third body; the period ratio between the two orbits is fixed to 28 . This leads to a semi-major axis ratio $\alpha=0.1$. The initial eccentricities of the orbits are fixed initially to $e_{\text {in }}=0.08$ and $e_{\text {out }}=0.27$, and the longitude of periastron of the outer orbit with respect to the periastron of the inner orbit is fixed initially to $270^{\circ}$.

Defined this way, our problem is scale-invariant. Our time basis will the orbital period of the inner orbit. As usual in HJS and MVS runs, we first fix the time-step to $1 / 20$ of that time basis. The main parameter we wish to describe here is the eccentricity of the inner orbit, as a function of the mutual inclination between the two orbits, as this is the most critical evolution. Figure 4 shows this evolution, as computed with the HJS2 integrator over $2 \times 10^{4}$ revolutions of the inner orbit, for an initial mutual inclination of $i=20^{\circ}$. It can be directly compared to the corresponding integration with the same initial parameters given in Fig. 2 from Krymolowski \& Mazeh (1999) (We only display the evolution over $2 \times 10^{4}$ revolution instead of $4 \times 10^{4}$ in order to better visualize the evolution). Figure 4 appears virtually identical to their direct integration of Newton's equations with a Burlish \& Stoer method. We confirm that for such moderate inclinations, the eccentricity of the inner orbit is subject to a superposition of two modulations: a large amplitude, long-term one (period $\simeq 380$ inner revolutions), and a smaller amplitude, more rapid one (period $\simeq 80$ inner revolutions). The mutual inclination $i$, initially fixed at $20^{\circ}$, appears to rapidly fluctuate around this value with an amplitude less than $0.2^{\circ}$, so twe do not display it here.

We get the same result as the direct integration by Krymolowski \& Mazeh (1999), thus confirming the validity of the HJS integration. However, the main difference is that we use here a very large time-step compared to the one needed for direct integrations. As a consequence, it only takes us a few minutes of CPU time to perform the integration, compared to several hours with direct methods.

As a further confirmation, we also perform the same integration with the HJS4 (fourth order) integrator. We do not

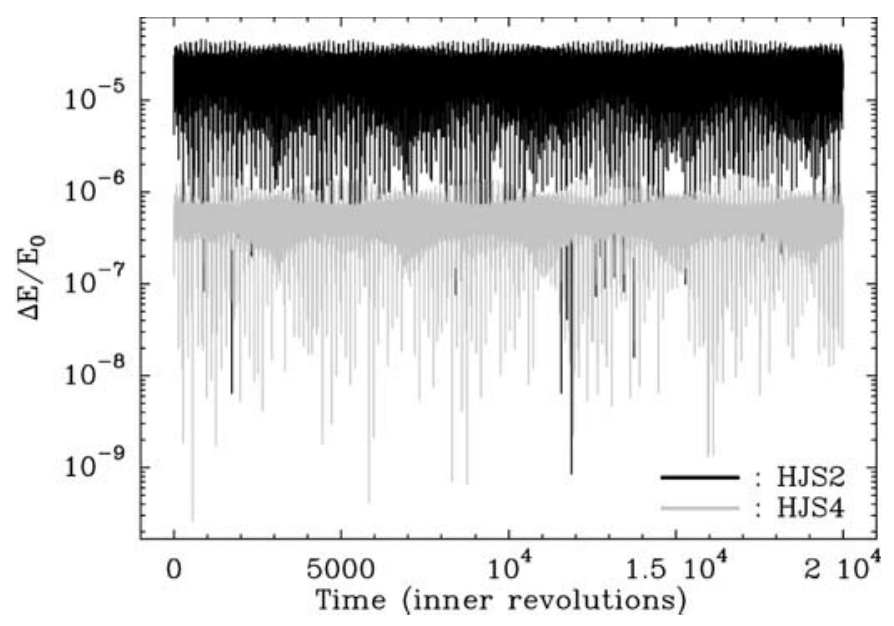

Fig. 5. Relative energy error with respect to the initial value as a function of time, for the integration corresponding to Fig. 4, in the cases of the use of the HJS2 integrator (black) and of the HJS4 integrator (grey).

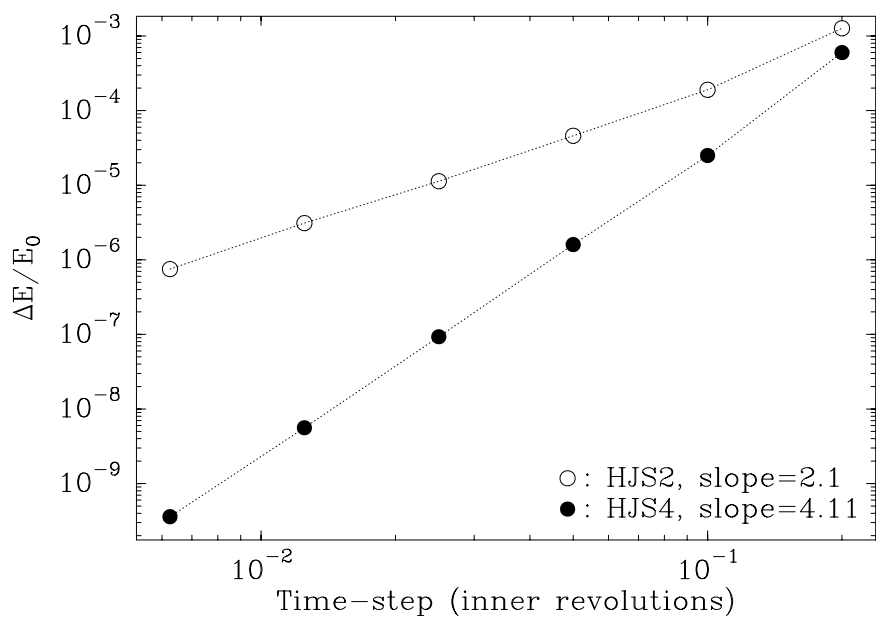

Fig. 6. Maximum relative energy error over several integrations of the triple system with $i=20^{\circ}$ over $2 \times 10^{4}$ inner revolutions, as a function of the time-step assumed, for the HJS2 and the HJS4 integrators. The empiric slopes confirm that the HJS2 method is of second order and the HJS4 of fourth order.

display here the corresponding result for the evolution of $e_{\text {in }}$, as it appears to exactly match Fig. 4. It is more interesting to check the energy preservation. Checking energy preservation was of no use in our circumbinary disk test, as the two massive bodies moved on a fixed orbit. Conversely, this is here of interest, as we have three massive bodies. Figure 5 shows the relative energy error with respect to the initial value, as a function of time, for the two (HJS2 and HJS4) integrations. In the HJS2 run, it appears to range between $10^{-9}$ and a few $10^{-5}$, which is equivalent to what Krymolowski \& Mazeh (1999) get in their integrations. In the HJS4 integration, the relative energy error now remains $\lesssim 10^{-6}$, for a roughly doubled computing time with respect to the HJS2 integration.

Additional checking can be achieved if we now let the time-step vary. This is illustrated in Fig. 6, which displays the maximum relative energy error quoted over the $2 \times 10^{4}$ inner revolution integrations, for various time-steps and with both 


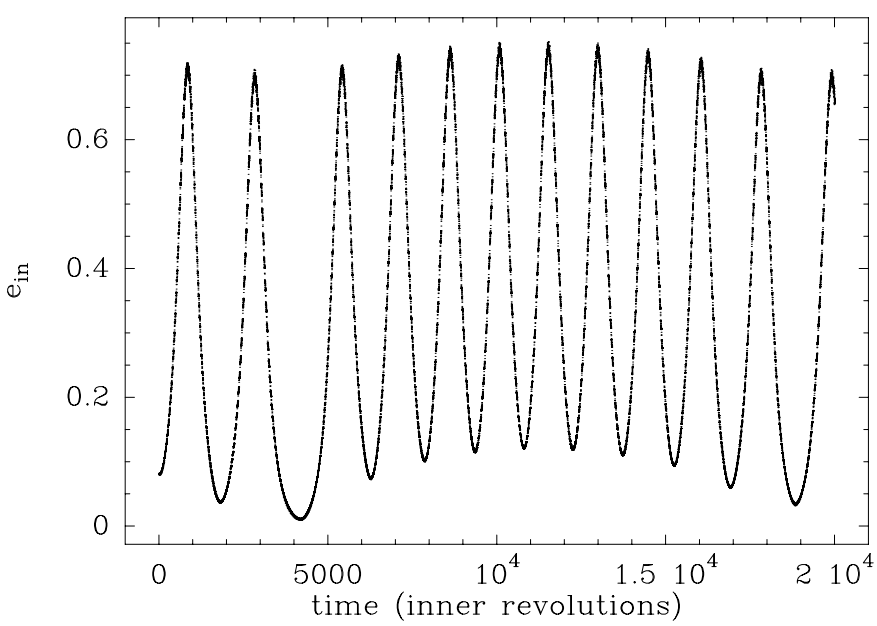

Fig. 7. Same as Fig. 4, but for an initial mutual inclination $i=60^{\circ}$. The eccentricity now undergoes large amplitude variations.

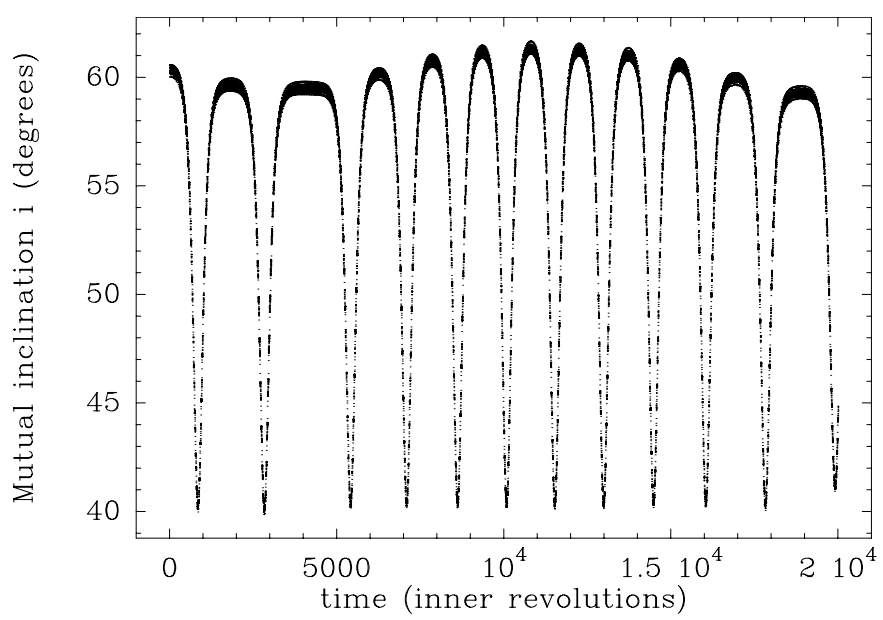

Fig. 8. Evolution of the mutual inclination $i$ for the run corresponding to Fig. 7. The inclination is subject to strong changes in phase with those of $e_{\text {in }}$.

integrators, in logarithmic scale. As a matter of fact, we get a slope close to 2 for the HJS2 integrator, and close to 4 for the HJS4 integrator.

Figure 7 displays the evolution of $e_{\text {in }}$ under the same conditions as Fig. 4, but with an initial inclination $i=60^{\circ}$. It can again be directly compared to the corresponding figure (Fig. 4) from Krymolowski \& Mazeh (1999). It exactly matches their direct integration of Newton's equation.

The secular evolution of $e_{\text {in }}$ is here very different from the $i=20^{\circ}$ case. The inner eccentricity is subject to drastic changes that bring it periodically as high as $\sim 0.7$. Figure 8 shows the corresponding evolution of the mutual inclination $i$ between the two orbits. Contrary to the $i=20^{\circ}$ case, $i$ is not stable here. It periodically drops down to $\sim 40^{\circ}$, and these events appear in phase with the high $e_{\text {in }}$ episodes.

This behavior is actually not surprising; obtaining it is in fact a validity test for the HJS method. It is known as the Kozai Resonance, initially described by Kozai (1962) for comets in the Solar System. In the Solar System, it concerns bodies with initial high inclinations and moderate eccentricities with respect to the mid-plane of the Solar System. Under the effect of secular planetary perturbations (mainly arising from Jupiter), the orbit is subject to an evolution that drives it periodically to lower inclination but very high eccentricity, the semi-major axis remaining constant. The combined evolution of inclination and eccentricity is due to the secular conservation of the vertical component of the orbital angular momentum $\Theta=\sqrt{a\left(1-e^{2}\right)} \cos i$ (thanks to rotational invariance). Hence if $i$ decreases, then $e$ must increase to keep $\Theta$ constant. This periodic evolution is characterized by stopping of the precession rate of the argument of perihelion $\omega$, which librates around $90^{\circ}$ or $-90^{\circ}$. As pointed out by Bailey et al. (1992), this mechanism is responsible for the origin of most Sun-grazer comets in our Solar System, in particular those of the Kreutz group.

The Kozai resonance is due to the secular part of the interaction Hamiltonian of the restricted circular three-body system (see diagrams in Kozai 1962). It is therefore very generic and is active as soon as one perturbing planet is present. The behavior reported in Figs. 7 and 8 is actually a Kozai resonance, although it concerns here a general (unrestricted) three-body system. Harrington (1968) first claimed that such a mechanism should be expected in a hierarchical three-body system with high mutual inclination, and Söderhjelm (1982) showed that it is active as soon as $i \gtrsim 40^{\circ}$.

In cometary dynamics, the Kozai resonance modifies the orbit of the particle, not that of the perturbing planet. It can appear surprising that here only the inner orbit is affected. As pointed out in Beust et al. (1997), the more affected orbit in the Kozai resonance is always the one with the smaller angular momentum. Here the angular momentum of the outer orbit is larger than that of the inner orbit.

This evolution may have some important observational outcomes. Some systems have indeed been observed with a significant eccentricity of the short-period inner binary, while it should have been circularized a long time ago (Mazeh 1990). In the most extreme cases, the eccentricity increase of the inner orbit could lead to a collision between its two stellar components, and thus to an instability of the system. However, for short period binaries, tidal effects between the components of the inner binary can severely dampen the modulation of $e_{\text {in }}$, thus stabilizing the system (Söderhjelm 1984; Beust et al. 1997).

As an extreme application case of this dynamical evolution, Fig. 9 displays the evolution of $e_{\text {in }}$ for the TY Coronae Australis (TY Cra) system. This system consists of a central, close eclipsing binary (semi-major axis $0.066 \mathrm{AU}$; masses 3.0 and $\left.1.6 M_{\odot}\right)$ surrounded by a tertiary component $\left(\sim 1.26 M_{\odot}\right)$ orbiting the binary at $\sim 1.5 \mathrm{AU}$ (Corporon et al. 1996). The mutual inclination between the two orbits is close to $80^{\circ}$. In Beust et al. (1997), we investigated the dynamics of this system, showing that a strong Kozai resonance should be expected if no tidal effect was active within the inner binary. This is actually confirmed by Fig. 9, which shows the secular evolution of $e_{\text {in }}$ under such conditions. We note a drastic Kozai resonance, the peak eccentricity being $\sim 0.9956$ ! Meanwhile, the mutual inclination $i$ is subject to sharp drops by more than $40^{\circ}$. It is worth noticing that even in such extreme cases the HJS 


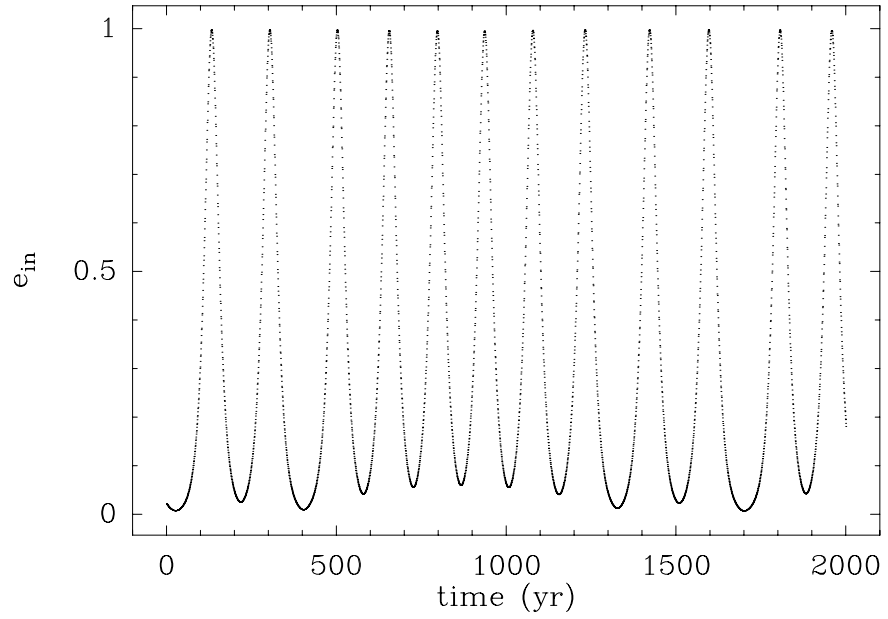

Fig. 9. Evolution of the inner eccentricity of the TY Cra system (without tidal effects) as computed with the HJS integrator. A strong Kozai resonance is visible.

integrator is still efficient: the maximum relative energy error during the eccentricity peaks does not exceed a few $10^{-4}$. This is nevertheless larger than in Fig. 5, but still acceptable.

Rauch \& Holman (1999) note that MVS-like algorithms may present some instability when handling very eccentric orbits, with relative energy errors that can reach unity! Even if our energy error still remains significantly smaller, our integration suffers presumably suffers here from a similar instability. Some regularization techniques are given by Rauch \& Holman (1999) and could be tested in the present case, be we did not investigate this question as it is beyond the scope of the present paper.

\subsection{A more complex example: The GG Tauri system}

GG Tauri (GG Tau) is a prototype example of a young, premain sequence multiple stellar system. It is located at $140 \mathrm{pc}$ and consists of a close $\left(\sim 0.3^{\prime \prime}\right)$ central binary, and of a more distant pair $\left(\sim 1.4^{\prime \prime}\right)$ located $\sim 10^{\prime \prime}$ to the south (Guilloteau et al. 1999). Whether these two binaries are actually gravitationally bound is still an open question, but it is highly probable. From combined dynamic and photometric studies it was possible to constrain the masses of the individual components of this system: the central binary is more massive $\left(\sim 0.6\right.$ and $\left.\sim 0.7 M_{\odot}\right)$ than the outer one $\left(\sim 0.12\right.$ and $\left.\sim 0.04 M_{\odot}\right)$ (White et al. 1999; Simon et al. 2000). Actually nearly all characteristics of the orbit of the inner binary can be deduced from the observations (Roddier et al. 1996), while for the two other orbits (the orbit of the outer binary and the mutual orbit between the two binaries) only spatial separations can be deduced.

The central binary is now well known to be surrounded by a dusty and gaseous circumbinary disk that was proved to be in Keplerian rotation around it. As expected for a circumbinary disk, the disk is truncated inwards, with a very sharp inner edge at $180 \mathrm{AU}$. More surprisingly, the disk also appears somehow truncated outwards. Actually, the dust data reveal that almost $70 \%$ of the material is confined in a sharp edged ring-like structure extending between 180 and $260 \mathrm{AU}$, while the $\mathrm{CO}$ data show that the rest of the material extends up to $800 \mathrm{AU}$ or more (Guilloteau et al. 1999).

From a dynamical point of view, the inward truncation of the disk is obviously due to a classical interaction with the central binary like the one we described above, the location of the inner edge appearing as a constraining parameter to the orbit of the binary. The outer shape appears more puzzling to interpret, but it is tempting to attribute it to an interaction with the outer binary. This issue needs nevertheless to be more precisely addressed; the HJS integrator appears indeed well suited for that.

This study will be the subject of a forthcoming paper (Beust $\&$ Dutrey 2003). We may nevertheless summarize here a few results. Figure 10 first shows one evolution of the system over $1.5 \times 10^{7} \mathrm{yrs}$. In this simulation, the four massive bodies were given their corresponding masses, and the orbits were chosen in order to match at $t=0$ the presently observed conditions. Of course there were free parameters, but the major ones are the periastron value $q$ of the mutual orbit between the two binaries, and the inclinations $i$ and $i^{\prime}$ between that orbit and those of the individual binaries ( $i$ with respect to the inner binary and $i^{\prime}$ with the outer one). The evolution displayed in Fig. 10 corresponds to $q=833 \mathrm{AU}, i=20^{\circ}$ and $i^{\prime}=150^{\circ}$. The disk is simulated as 50000 test particles initially chosen orbiting the central binary between 150 and $1200 \mathrm{AU}$, i.e., much wider than the observed disk. With these parameters, the gravitational pull between the binaries at each periastron passage progressively sculpts the disks and finally the residual disk appears very close to the observed one. This will be fully detailed in Beust \& Dutrey (2003). Of course the evolution depends on the initial orbital parameters. If $q$ is too small, the disk is entirely destroyed, and if it is too large, the remaining disk is too thick to match the observation; if $i$ is large, we initiate a Kozai resonance between the orbit of the inner binary and the wide orbit. This evolution brings the eccentricity of the inner binary to large values, and this erodes the disk from inside much further out than the observed $180 \mathrm{AU}$ inner edge.

$i^{\prime}$ values around $150^{\circ}$ (i.e. a retrograde orbit for the outer binary with respect to the mutual orbital motion) appear to be the only range of values that ensures the stability of the orbit of the outer binary. It can be seen in Fig. 10 that the separation between the components of the outer binary is not small compared to the size (and the periastron) of the wide orbit. Hence the basic hypothesis of hierarchical motion is marginally fulfilled. This may result in an instability of the outer binary, its orbit possibly being tidally disrupted. The HJS integrations revealed that retrograde orbits for the outer binary are much more stable than prograde and perpendicular ones. This comes from the fact that if the orbit is retrograde, then at each periastron passage between the two binaries, the orbital motion between the two components of the outer binary is observed to be more rapid from the inner binary. Consequently the perturbations are better averaged over the orbital motions, and the whole system is more stable. In the opposite limiting case (prograde orbit), the outer binary is close to synchronous revolution of its orbital motion with respect to the inner binary at each periastron passage. The tidal pull is subsequently very strong and the outer binary is unstable. 

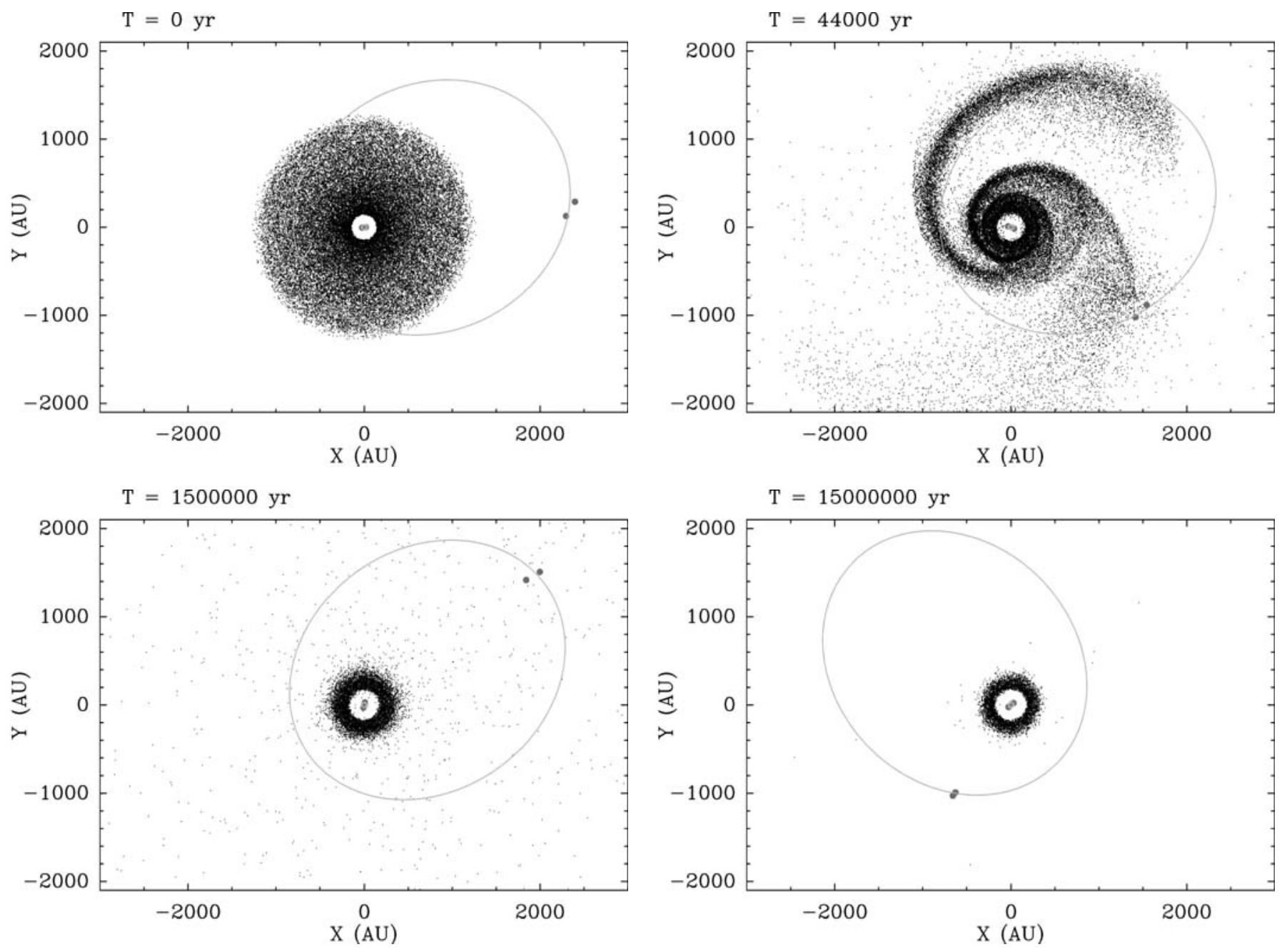

Fig. 10. Upper view of the four-body GG Tau system with its circumbinary disk, as simulated with the HJS integrator. The first plot a) shows the initial disk together with the four stars and the wide orbit. The following plots show the evolution of the system after b) $44000 \mathrm{yr}$, i.e., after the first periastron passage between the two binaries, after c) $1.5 \times 10^{6} \mathrm{yr}$, i.e., something comparable to the present age of GG Tau, and after d) $1.5 \times 10^{7} \mathrm{yr}$, at the end of the simulation. We see how the disk is progressively sculpted by the successive periastron passages. The residual disk closely matches the observed one. We also note the secular precession of the periastron of the wide orbit over the simulation time.

\subsection{The Castor sextuple system}

As a final test for the HJS integrator, we briefly investigate here the dynamics of the remarkable sextuple system Castor $(\alpha \mathrm{Gem}) . \alpha \mathrm{Gem}$ itself is a visual binary (Castor A and B) with a present angular separation of 3.9". This corresponds to a physical separation of $\sim 58 \mathrm{AU}$, thanks to the parallax measurement by Hipparcos $(66.90 \pm 0.63$ mas; Torres \& Ribas 2002). The orbit between Castor A and B is well known (Heintz 1988), with an orbital period of of $467 \mathrm{yr}$. Each of these components is itself a single-lined spectroscopic binary, with orbital periods of a few days. A more distant, eclipsing binary (Castor C, or YY Gem), is seen at $71^{\prime \prime}$. It is located at the same distance as the rest of the system, and it is therefore probably bound to the whole system. YY Gem is a double-lined spectroscopic binary with a period of 0.814 day. The whole system is thus sextuple.

As noted by Torres \& Ribas (2002), the individual masses of the six individual components can be deduced from combined observations and modeling. Most of the characteristics of the different orbits constituting the system are also known (Torres \& Ribas 2002).

The summary of known dynamical information about the Castor system is given in Table 1. These data are extracted from Torres \& Ribas (2002) and Heintz (1988), and converted using the Hipparcos parallax. The hierarchical system consists thus of six stars and five orbits. From here on, we adopt the following numbering convention: orbits \#1, \#2 and \#3 will be the internal orbits of binaries A, B and C, respectively. Orbit \#4 will be the orbit between Castor A and Castor B, and orbit \#5 will be wide orbit between Castor $\mathrm{C}$ and the rest of the system (Castor A+B) (see Table 1).

Of course many orbital parameters are still unknown, despite a large amount of observational data. The best known orbit is orbit \#4, the orbital motion of Castor B with respect to Castor A, as the motion of both binaries has been now followed for several tens of years (Heintz 1988). Conversely, the motion of Castor $\mathrm{C}$ around Castor $\mathrm{A}+\mathrm{B}$ (orbit \#5) is poorly known, as only a projected separation of $1060 \mathrm{AU}$ can be inferred. 
Table 1. Summary of observational characteristics of the Castor system. The orbital inclinations are given with respect to the plane of the sky.

\begin{tabular}{lcccccc}
\hline \hline $\begin{array}{l}\text { Mass information: } \\
\text { Component }\end{array}$ & $\mathrm{Aa}$ & $\mathrm{Ab}$ & $\mathrm{Ba}$ & $\mathrm{Bb}$ & $\mathrm{Ca}$ & $\mathrm{Cb}$ \\
\hline Mass $\left(M_{\odot}\right)$ & 2.27 & 0.45 & 1.79 & 0.32 & 0.60 & 0.60 \\
\hline \hline Orbital information: & \multicolumn{7}{c}{$\mathrm{C}-\mathrm{Bb}-\mathrm{Bb}$} & $\mathrm{Ca}-\mathrm{Cb}$ & $\mathrm{A}-\mathrm{B}$ & $\mathrm{AB}-\mathrm{C}$ & \\
Orbit & $\mathrm{Aa}-\mathrm{Bb}$ & $\mathrm{Ba}$ & & \\
Numbering convention & $\# 1$ & $\# 2$ & $\# 3$ & $\# 4$ & $\# 5$ & \\
\hline Semi-major axis (AU) & 0.12 & 0.0514 & 0.0181 & 101.75 & $10^{3(*)}$ & \\
Eccentricity & 0.4992 & 0.0 & 0.0 & 0.343 & $?$ & \\
Inclination (deg.) & 28. & 90. & 86.3 & 114.5 & $?$ & \\
Longitude of node & $?$ & $?$ & $?$ & 41.3 & $?$ & \\
Argument of periastron & $?$ & $?$ & $?$ & 249.5 & $?$ & \\
Mean anomaly (deg.) & $?$ & $?$ & $?$ & 28.29 & $?$ & \\
\hline
\end{tabular}

(*) Only the present separation of $1060 \mathrm{AU}$ is known.

This implies an orbital period $\gtrsim 14000 \mathrm{yr}$, which makes it hard to detect orbital motion on a short time basis.

The HJS integrator may be a well-suited tool for studying the long term dynamics of such a system, as it consists of several orbits of very different sizes. Although a lot of dynamical information is known about the system, there are still many parameters unknown (Table 1), and it is virtually impossible to explore the whole parameter space. As yet, the only global study of the dynamics of the Castor system was made by Anosova et al. (1989) on a statistical trial basis, treating the three binaries as individual point masses. Anosova et al. (1989) concluded nevertheless that the sextuple system was gravitationally bound, and that the wide orbit (orbit \#5) was probably eccentric.

Here we want to try to compute the dynamical evolution of the sextuple system, as a final test of the HJS integrator. The test is interesting because the dynamics of the system involves very different time-scales, ranging from less than one day for the orbital motion of the individual binaries to more than $10^{4}$ yrs for orbit \#5. Hence we will need to adopt a small time-step in order to correctly compute orbits \#1,2 and 3, but to carry out the integration over a long enough time basis, in order to see the secular evolution of orbits \#4 and \#5.

We only describe here one computed evolution as an illustration of the capabilities of the HJS integrator. Exploring the whole parameter space of initial conditions that are not fixed in Table 1 is beyond the scope of the present paper. Hence we take the initial conditions given in Table 1, assuming a semimajor axis of $1060 \mathrm{AU}$ and an eccentricity of 0.5 for orbit \#5. All other unknown parameters in Table 1 are set to 0.0 at the beginning of the simulation. We expect the most important unknown parameters to be those concerning orbit \#5. Depending on the mutual inclination between subsequent orbits, we expect to find Kozai resonances between some of the orbits.

The integration we present here was carried out for $10^{6} \mathrm{yrs}$, using a time-step of $1 / 20$ of the smallest orbit (\#4), i.e. 1 hour. This took approximatively one week of CPU on a single

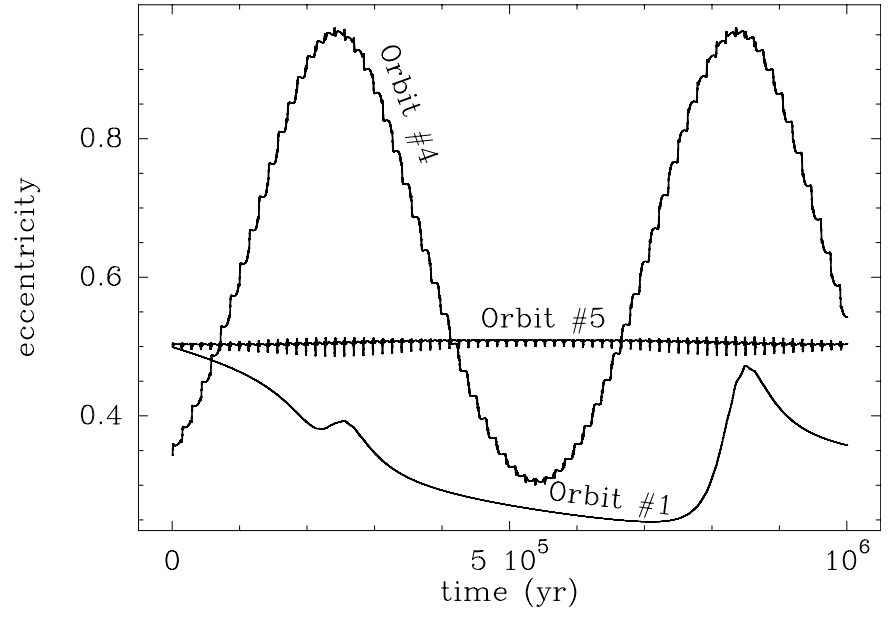

Fig. 11. Evolution of the eccentricities of orbit \#1, 4 and 5 of the Castor system as computed with the HJS integrator. Orbits \#2 and 3 are not shown as their eccentricities remain permanently very low $\left(\lesssim 10^{-4}\right)$.

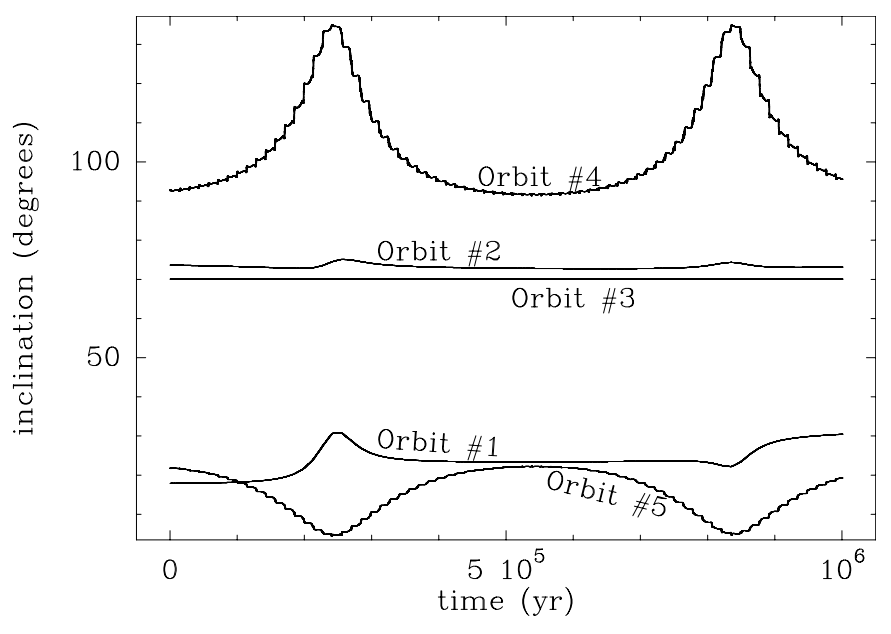

Fig. 12. Evolution of the inclinations of all orbits of the Castor system as computed with the HJS integrator. The inclinations are computed with respect to the invariable plane of the whole system (perpendicular to its angular momentum).

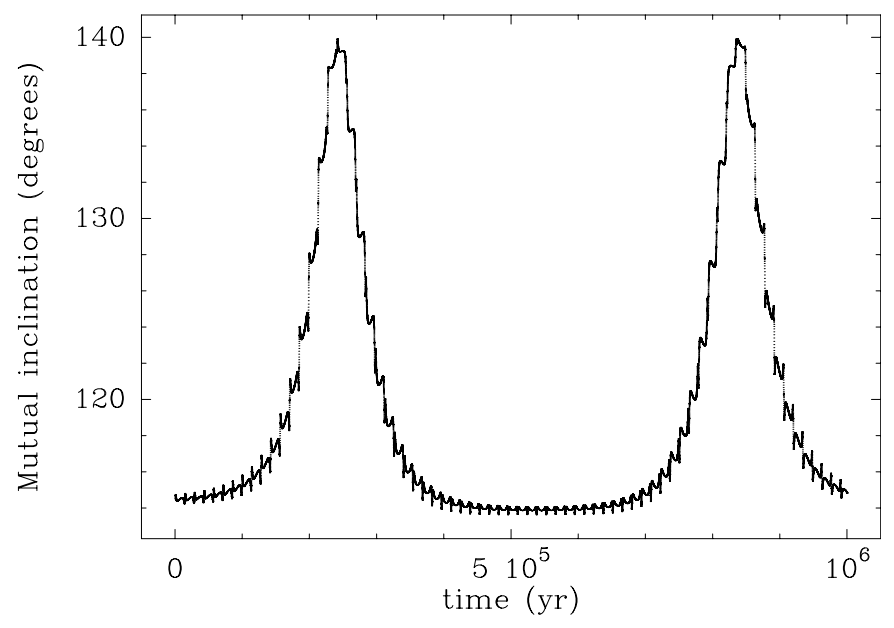

Fig. 13. Evolution of the mutual inclination of orbit \#4 with respect to orbit \#5, as computed with the HJS integrator. 


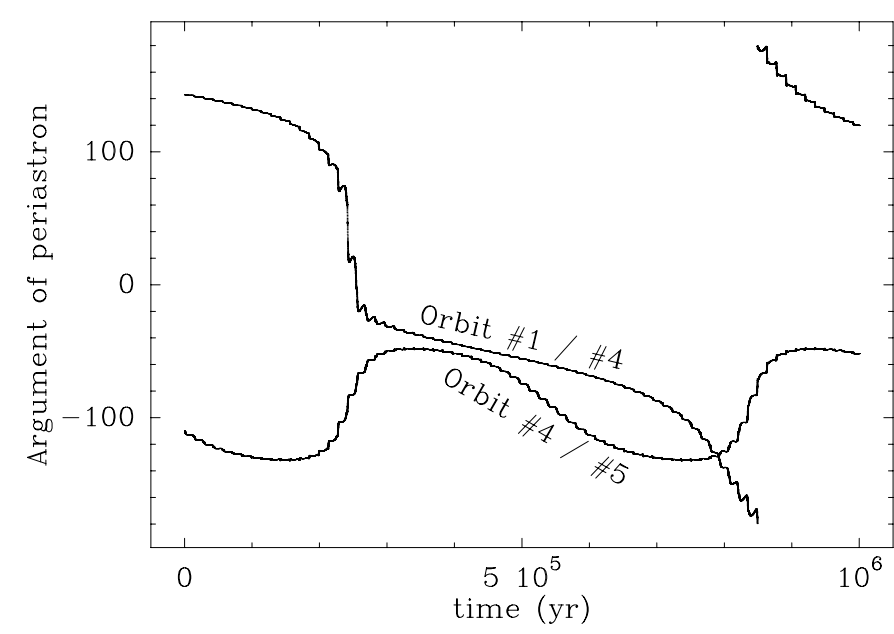

Fig. 14. Same as Fig. 13, but for the argument of periastron of orbit\#4 with respect to the plane of orbit\#5, and similarly for orbit\#1 with respect to orbit \#4.

IBM power3+ processor. The maximum relative energy error over the whole integration was $\sim 10^{-7}$.

Figure 11 shows the computed evolution of the orbital eccentricities of orbits \#1, 4 and 5. The eccentricities of orbits \#2 and 3 remain very small and do not need to be displayed here. Figure 12 shows the evolution of the inclinations of all orbits, as computed with respect to the invariable plane of the whole system.

We first note that orbits \#2 and \#3 are very stable. Their eccentricities remain very small and their inclinations are not subject to significant changes; these orbits concern Castor B and $\mathrm{C}$, and they are the smallest ones, so that the inner motion of these two binaries is almost independent of the secular motion of the whole system. This is particularly the case for orbit \#3. We could have merged Castor $\mathrm{C}$ into a single body for the computing of the rest of the dynamics.

This is not the case for orbit\#1 (Aa-Ab), which is wider than orbits \#2 and \#3, and consequently clearly interacts with the global motion. The major changes actually concern orbits \#4 and \#5. We note a periodic modulation of the eccentricity of orbit\#4 together with synchronous changes of the inclinations of both orbits. There is obviously a Kozai resonance between them. This is confirmed by Fig. 13 which shows the evolution of the mutual inclination between the two orbits. As usual, we see in Fig. 12 that the inner orbit (\#4) is more affected by the outer (\#5), especially its eccentricity. This is due to the larger value of the angular momentum of orbit \#5 than of orbit \#4. However, the angular momentum ratio between these two orbits is only $\sim 2.7$, so that orbit\#5 is also affected by the Kozai resonance (Fig. 12).

However, with respect to standard behavior in Kozai resonance (see three body simulations above), we note in Figs. 1113 an additional, higher frequency modulation in the secular evolution of the eccentricities and inclinations of the orbits affected by the Kozai resonance (\#4 and \#5). This additional modulation is due to the interaction with the inner orbits (mainly orbit \#1), as the Kozai resonance reported here concerns three binaries instead of single bodies. Obviously the HJS integrator is very efficient to compute this dynamics.

Orbit \#1 (Castor Aa-Ab) is clearly affected by the interaction with the rest of the system. This is obvious in Figs. 11 and 12, which show significant eccentricity and inclination changes of that orbit. Despite the fairly large eccentricity modulation, this orbit is not trapped in a Kozai resonance with orbit \#5, i.e. the outer orbit just above it. This shows up in Fig. 14, which displays the secular evolution of the argument or periastron $\omega_{4,5}$ of orbit \#4 with respect to the plane orbit\#5, and that of $\omega_{1,4}$, which is the same for orbit\#1 with respect to orbit\#4. We note a libration of $\omega_{4,5}$ around $-90^{\circ}$, which is characteristic for a Kozai resonance. Conversely, $\omega_{1,4}$ is subject to a more or less regular precession, and is thus not confined around a fixed value.

Of course this dynamic depends on the initial conditions we choose; we could actually get different situations if we assumed different values. Exploring the whole parameter space is nevertheless beyond the scope of the present paper, as we only want to illustrate applications for the HJS orbit. We note nevertheless that finding a Kozai resonance between orbits \#4 and \#5 may appear not surprising, as the period ratio between them is $\sim 30$. Conversely, the period ratio between orbits \#1 and \#4 is $\sim 18500$. The typical period of the eccentricity modulation due to the Kozai resonance is $\sim P_{\text {out }}^{2} / P_{\text {in }}$, where $P_{\text {out }}$ and $P_{\text {in }}$ are the orbital periods of respectively the outer and inner orbits involved in the Kozai resonance (Söderhjelm 1982). For orbits \#4 and \#5 we derive a period of $4.3 \times 10^{5} \mathrm{yrs}$, which is somewhat shorter but comparable to the one appearing in Fig. 11. For orbits \#1 and \#4, the same estimate gives a period larger than $8 \times 10^{6} \mathrm{yrs}$, which is far larger than the time-span of our integration. Actually the shorter period perturbations on that orbit arising from all components of the system overcome the very long term Kozai modulation, making $\omega_{1,4}$ precess.

The absence of Kozai resonance between orbits \#1 and \#4 nevertheless does not prevent orbit\#1 from being significantly affected by the six-body system dynamics, while this is not the case for the closer orbits \#2 and \#3. This may actually explain why orbit\#1 is presently eccentric, which is not the case for orbits \#2 and \#3. These last two orbits may have been tidally circularized, while this is not the case for the wider orbit \#1.

\section{Conclusions and prospectives}

The HJS integrator is a modified MVS-like technique that permits the symplectic integration of $n$-body hierarchical systems with comparable masses. The different application examples described above show that is very well suited for the study of various dynamical configurations, even of high complexity. The gain in computing speed is at least one or two orders of magnitude with respect to conventional techniques where the first order hierarchical Keplerian motion is not built-in. In fact, the gain is comparable to the one provided by the MVS-like integrators designed for planetary system study.

The application examples presented in the present paper do not represent an exhaustive description of all the capabilities of the HJS integrator. There are many other problems to which it could apply, such as for example the dynamics of planets 
(S or P type) in binary or even multiple stellar systems. There are two major limitations to the HJS technique though. First, close encounters between two bodies are not permitted unless they are the only two members of one of the orbits followed in the integration. This difficulty was already encountered in the MVS technique for the study of the final (violent) stages of formation of planetary systems. Duncan et al. (1998) overcome this difficulty in their SyMBA integrator, but they need to split the Hamiltonian into three parts instead of two, and above all they use heliocentric coordinates, thus particularizing the central body. With the HJS integrator we move in the opposite direction, trying not to particularize any of the massive bodies. Hence the study of planetary system formation in multiple stellar systems could appear problematic with the HJS integrator if close encounters play a significant role. An intermediate technique dedicated to the study of planets in binary systems was recently developed by Chambers et al. (2002). Basically, the coordinates they introduce are almost equivalent to our generalized Jacobi ones, but all planets are treated on the same level. This allows them to handle close encounters, but consequently the Hamiltonian must be split into three parts; if the planetary orbits are treated on the same level, the full hierarchical structure is not respected. The Chambers et al. (2002) technique is identical to ours if only one planet is present in the binary system. If there are many planets, then the third part of the Hamiltonian does not cancel. The major consequence is that the second order acceleration formulas used in the code are much more complex; this renders the generalization to any configuration in a multiple stellar system more difficult. Chambers et al. (2002) use different explicit formulas (and thus different codes) depending whether type $S$ (orbiting one of the two stars) or type $\mathrm{P}$ (orbiting the binary) planets are considered. Of course this no longer applies if the stellar system harbors more than two stars. A valuable future evolution of the HJS integration would then be a possible synthesis of HJS and of the Chambers et al. (2002) technique. In order for this to be possible, the various bodies involved in the $n$-body system should be classified into three subsets instead of two: i) the "stars", i.e., the most massive bodies whose hierarchical structure must follow the rules defined in the present paper and which cannot undergo close encounters among themselves; ii) the "planets", i.e., less massive bodies that are supposed to orbit a given center of mass in the stellar system, with no other constraint on the hierarchical structure; iii) massless test particles that would be treated as usual.

Another limitation of the HJS technique is that the hierarchical structure of the system must be preserved along the integration. This is not in itself a very strong constraint, as in any hierarchical $n$-body system, whenever a Keplerian structure is broken, at least part of the bodies concerned are usually lost by the system within a very short time-scale. Hence even if the final escape motion is not well computed, the final result is the same.

Acknowledgements. All the computations presented in this paper were performed at the Service Commun de Calcul Intensif de l'Observatoire de Grenoble (SCCI).

\section{Appendix A: The kinetic energy in the HJS formalism}

In the matrix formulation introduced in the text, the kinetic energy can be written algebraically (with convenient product definition) as

$T={ }^{t} \boldsymbol{P} A \boldsymbol{P}$,

where $A$ is a diagonal matrix with $A_{i i}=1 /\left(2 m_{i}\right)$ for $1 \leq i \leq n$. $T$ is a function of the generalized Jacobi momenta $\boldsymbol{P}^{\prime}$. From $\boldsymbol{P}^{\prime}={ }^{t} M^{-1} \boldsymbol{P}$, we derive

$T={ }^{t} \boldsymbol{P}^{\prime} M A^{t} M \boldsymbol{P}^{\prime}={ }^{t} \boldsymbol{P}^{\prime} B \boldsymbol{P}^{\prime}$,

with $B=M A^{t} M$. Equation (14) will be proved if we can show that $B$ is also a diagonal matrix with $B_{i i}=1 /\left(2 m_{i}^{\prime}\right)$. This property is due to the hierarchical structure of the system. For each element $B_{i j}(1 \leq i, j \leq n)$, we can write

$B_{i j}=\sum_{k=1}^{n} M_{i k} A_{k k} M_{j k}=\sum_{k=1}^{n} \frac{M_{i k} M_{j k}}{2 m_{k}}$.

From this expression we first see that the matrix $B$ is symmetrical $\left(B_{i j}=B_{j i}\right)$. Then several cases occur:

1. $i=j=1$. In this case $M_{i k}=M_{j k}=m_{k} / m$ ( $m$ is the total mass), and

$$
B_{11}=\frac{1}{2 m^{2}} \sum_{k=1}^{n} m_{k}=\frac{1}{2 m}=\frac{1}{2 m_{1}^{\prime}} .
$$

2. $i=1$ and $j \neq 1$ (or the other way round). Then

$$
\begin{aligned}
B_{1 j} & =\sum_{k=1}^{n} \frac{m_{k}}{m} \frac{M_{j k}}{2 m_{k}}=\frac{1}{2 m} \sum_{k=1}^{n} M_{j k} \\
& =\frac{1}{2 m}\left(\sum_{k, \text { satellites of } j} \frac{m_{k}}{\mu_{j}}-\sum_{k, \text { centers of } j} \frac{m_{k}}{\eta_{j}}\right) \\
& =\frac{1}{2 m}\left(\frac{\mu_{j}}{\mu_{j}}-\frac{\eta_{j}}{\eta_{j}}\right)=0 .
\end{aligned}
$$

3. $i, j \neq 1$ and orbits $i$ and $j$ are foreign. In that case, for any body $k$, we cannot have simultaneously $M_{i k} \neq 0$ and $M_{j k} \neq$ 0 . Then we have obviously $B_{i j}=0$.

4. $i, j \neq 1$ and orbits $i$ and $j$ are not foreign. One orbit is thus inner to the other one. Let us consider that orbit $i$ is inner to orbit $j$. Then all the centers and satellites of orbit $i$ are taken from one of the two subsets (centers or satellites) of orbit $j$. Let us assume that they are taken from the satellites of orbit $j$. Then if $M_{i k} \neq 0$, necessarily body $k$ is a satellite of orbit $j$, and $M_{j k}=m_{k} / \mu_{j}$. We may then write

$$
\begin{aligned}
B_{i j} & =\sum_{k, \text { satellites of } i} \frac{1}{2 m_{k}} \frac{m_{k}}{\mu_{j}} \frac{m_{k}}{\mu_{i}}-\sum_{k, \text { centers of } j} \frac{1}{2 m_{k}} \frac{m_{k}}{\mu_{j}} \frac{m_{k}}{\eta_{i}} \\
& =\frac{1}{2 \mu_{j}}\left(\frac{\mu_{i}}{\mu_{i}}-\frac{\eta_{i}}{\eta_{i}}\right)=0 .
\end{aligned}
$$

Of course if the centers and satellites of orbit $i$ are taken from the centers of orbit $j$, we come to the same result. Finally $B_{i j}=0$ as soon as $i \neq j$. 
5. $i=j($ and $\neq 1)$. We write

$$
\begin{aligned}
B_{i i} & =\sum_{k=1}^{n} \frac{M_{i k}^{2}}{2 m_{k}} \\
& =\sum_{k, \text { satellites of } i} \frac{1}{2 m_{k}} \frac{m_{k}^{2}}{\mu_{i}^{2}}+\sum_{k, \text { centers of } i} \frac{1}{2 m_{k}} \frac{m_{k}^{2}}{\eta_{i}^{2}} \\
& =\frac{1}{2}\left(\frac{\mu_{i}}{\mu_{i}^{2}}-\frac{\eta_{i}}{\eta_{i}^{2}}\right)=\frac{1}{2} \frac{\eta_{i}+\mu_{i}}{\eta_{i} \mu_{i}}=\frac{1}{2 m_{i}^{\prime}} .
\end{aligned}
$$

The matrix $B$ appears then to verify the desired property and Eq. (14) is proved.

\section{Appendix B: The Hamiltonian splitting in the HJS method}

We recall the way we split the Hamiltonian of the $n$-body problem in the HJS method.

$H_{A}=\sum_{k=2}^{n}\left(\frac{p_{k}^{\prime 2}}{2 m_{k}^{\prime}}-\frac{G \mu_{k} \eta_{k}}{r_{k}^{\prime}}\right) ;$

$H_{B}=\sum_{k=2}^{n} \frac{G \mu_{k} \eta_{k}}{r_{k}^{\prime}}-\sum_{1 \leq i<j \leq n} \frac{G m_{i} m_{j}}{r_{i j}}$.

We want to prove that $H_{B} \ll H_{A}$. Note that the first sum in $H_{B}$ extends over the orbits, while the second sum extends over the bodies. The key point here is that given one pair of bodies $(i, j)$, there is one and only one orbit $k$ for which one of the bodies is a satellite in orbit $k$ and the other one a center in the same orbit. There is one because if there was no orbit concerned, it would be possible to split the $n$-body system into two distinct, noninteractive systems, which is obviously not the case. At the top of the hierarchical tree, there is necessarily a first orbit that concerns all the bodies. If for the two bodies under consideration, one is a satellite of that orbit and the other one a center, then we have found the orbit we are seeking. If this is not the case, then both bodies are part of one of the two subsets (centers or satellites) of the first orbit. It is then possible to re-initiate the same argumentation with the subset concerned. Finally, we necessarily find in the tree an orbit with the desired property. Given this result, it is then possible to rewrite the second sum of $H_{B}$ as a sum over the orbits:

$H_{B}=\sum_{k=2}^{n}\left(\frac{G \mu_{k} \eta_{k}}{r_{k}^{\prime}}-\sum_{\substack{i, \text { centers of } k \\ j, \text { satellites of } k}} \frac{G m_{i} m_{j}}{r_{i j}}\right)$.

Now, we make use of the assumption that the successive orbits have very different sizes. Given one orbit $k$, all orbits inner to it concern either the satellites of orbit $k$, either its centers. These orbits are much smaller than orbit $k$, showing that the sizes of the satellites and centers groups of orbit $k$ are much smaller than the size of the orbit itself. Hence, given one center $i$ and one satellite $j, \boldsymbol{r}_{i j}$ is close to $\boldsymbol{r}_{k}^{\prime}$. We may then write

$\boldsymbol{r}_{i j}=\boldsymbol{r}_{k}^{\prime}+\boldsymbol{\epsilon}_{i j}$ with $\epsilon_{i j} \ll r_{k}^{\prime}$. We also have

$$
\sum_{\substack{i, \text { centers of } k \\ j, \text { satellites of } k}} m_{i} m_{j} \boldsymbol{\epsilon}_{i j}=0,
$$

because $\boldsymbol{r}_{k}^{\prime}$ is defined from the barycenters of the satellites and of the centers or orbit $k$. We write

$$
\frac{1}{r_{i j}}=\frac{1}{\sqrt{r_{k}^{\prime 2}+\boldsymbol{r}_{k}^{\prime} \cdot \boldsymbol{\epsilon}_{i j}+\epsilon_{i j}^{2}}} \text {. }
$$

Now, we make a classical expansion of $1 / r_{i j}$ :

$\frac{1}{r_{i j}}=\frac{1}{r_{k}^{\prime}}\left[1-\frac{1}{2} \frac{\boldsymbol{r}_{k}{ }_{k} \cdot \boldsymbol{\epsilon}_{i j}}{{r_{k}^{\prime}}^{2}}+O\left(\frac{\epsilon^{2}}{{r_{k}^{\prime}}^{2}}\right)\right]$,

where $\epsilon$ is a characteristic size for the various $\epsilon_{i j}$ 's. We perform the sum now over all couples $(i, j)$. We get

$$
\begin{aligned}
& \sum_{\substack{i, \text { centers of } k \\
j, \text { satellites of } k}} \frac{G m_{i} m_{j}}{r_{i j}}=\sum_{\substack{i, \text { centers of } k \\
j, \text { satellites of } k}}\left[\frac{G m_{i} m_{j}}{r_{k}^{\prime}}\right. \\
& \left.\quad-\frac{G m_{i} m_{j}}{2} \frac{\boldsymbol{r}^{\prime}{ }_{k} \cdot \boldsymbol{\epsilon}_{i j}}{r_{k}^{\prime 3}}+\frac{1}{r_{k}^{\prime}} O\left(\frac{\epsilon^{2}}{r_{k}^{\prime 2}}\right)\right] \\
& =\frac{G \mu_{k} \eta_{k}}{r_{k}^{\prime}}-\frac{G}{2 r_{k}^{\prime 3}} \boldsymbol{r}_{k}^{\prime}{ }_{\substack{i, \text { centers of } k \\
j, \text { satellites of } k}} m_{i} m_{j} \boldsymbol{\epsilon}_{i j}+\frac{1}{r_{k}^{\prime}} O\left(\frac{\epsilon^{2}}{r_{k}^{\prime 2}}\right) \\
& =\frac{G \mu_{k} \eta_{k}}{r_{k}^{\prime}}+\frac{1}{r_{k}^{\prime}} O\left(\frac{\epsilon^{2}}{r_{k}^{\prime 2}}\right) .
\end{aligned}
$$

The first term cancels with the first one in $H_{B}$ so that the part of $H_{B}$ corresponding to orbit $k$ is just $\left(1 / r_{k}^{\prime}\right) O\left(\epsilon^{2} / r_{k}^{\prime 2}\right)$. As the corresponding part of $H_{A}$ (Keplerian Hamiltonian) if of order $1 / r_{k}^{\prime}$, we get by summation that $H_{B} \ll H_{A}$ to second order.

\section{Appendix C: The HJS package}

HJS was built as a superset of the popular SWIFT package (Levison \& Duncan 1994). The routines constituting HJS are publicly available via http://www-laog.obs.ujf-grenoble.fr/ beust/hjs.html. They must must be added to the SWIFT package and compiled with it. Instruction for compilation and for running are given via the same URL.

\section{References}

Anosova, Zh. P., Orlov, V. V., \& Chernyshev, M. V. 1989, Soviet Astron. Lett., 15, 237

Artymowicz, P., \& Lubow, S. H. 1994, ApJ, 421, 651

Artymowicz, P., \& Lubow, S. H. 1996, ApJ, 467, L77

Bailey, M. E., Chambers, J. E., \& Hahn, G. 1992, A\&A, 257, 315

Bate, M. R. 2000, MNRAS, 314, 33

Beust, H., Corporon, P., Siess, L., Forestini, M., \& Lagrange, A.-M. 1997, A\&A, 320, 478

Beust, H., \& Dutrey, A. 2003, in preparation

Chambers, J. E. 1999, MNRAS, 304, 793

Chambers, J. E., \& Murison, M. A. 2000, AJ, 119, 425 
Chambers, J. E., Quintana, E. V., Duncan, M. J., \& Lissauer, J. J. 2002, AJ, 123, 2884

Close, L. M., Dutrey, A., Roddier, F., et al. 1998, ApJ, 499, 883

Corporon, P., Lagrange, A.-M., \& Beust, H. 1996, A\&A, 310, 228

Duncan, M. J., Levison, H. F., \& Lee, M. H. 1998, AJ, 116, 2067

Duvert, G., Dutrey, A., Guilloteau, S., et al. 1998, A\&A, 332, 867

Guilloteau, S., Dutrey, A., \& Simon, M. 1999, A\&A, 348, 570

Harrington, R. S. 1968, AJ, 73, 190

Heintz, W. D. 1988, PASP, 100, 834

Koerner, D. W., Jensen, E. L. N., Cruz, K. L., Guild, T. B., \& Gultekin, K. 2000, ApJ, 533, L37

Koresko, C. D. 1998, ApJ, 507, L145

Kozai, Y. 1962, AJ, 67, 591

Krymolowski, Y., \& Mazeh, T. 1999, MNRAS, 304, 720

Levison, H. F., \& Duncan, M. J. 1994, Icarus, 108, 18

Levison, H. F., \& Duncan, M. J. 2000, AJ, 120, 2117

Mazeh, T. 1990, AJ, 99, 675
Mazeh, T., \& Shaham, J. 1977, ApJ, 213, L17

Mazeh, T., \& Shaham, J. 1977, A\&A, 77, 145

Quintana, E. V., Lissauer, J. J., Chambers, J. E., \& Duncan, M. J. 2002, ApJ, 576, 982

Rauch, K. P., \& Holman, M. 1999, AJ, 117, 1087

Roddier, C., Roddier, F., Northcott, M. J., Graves, J. E., \& Jim, K. 1996, ApJ, 463, 326

Saha, P., \& Tremaine, S. 1992, AJ, 104, 1633

Simon, M., Dutrey, A., \& Guilloteau, S. 2000, ApJ, 545, 1034

Söderhjelm, S. 1982, A\&A, 107, 54

Söderhjelm, S. 1984, A\&A, 141, 232

Stapelfeldt, K. R., Krist, J. E., Ménard, F., et al. 1998, ApJ, 502, L65

Torres, G., \& Ribas, I. 2002, ApJ, 567, 1140

White, R. J., Ghez, A. M., Reid, N. I., \& Schultz, G. 1999, ApJ, 520, 811

Wisdom, J., \& Holman, M. 1991, AJ, 102, 1528

Yoshida, H. 1990, Phys. Lett. A, 150, 262 